\title{
Effective eddy diffusivities inferred from a point release tracer in an eddy-resolving ocean model
}

\author{
Mei-Man Lee, A. J. George Nurser, Andrew C. Coward, and \\ Beverly A. de Cuevas \\ National Oceanography Centre, Southampton, UK.
}

October 6, 2008

accepted in JPO 


\begin{abstract}
This study uses tracer experiments in a global eddy-resolving ocean model to examine two diagnostic methods for inferring effective eddy isopycnic diffusivity from point release tracers. The first method is based on the growth rate of the area occupied by tracers (the equivalent variance). During the period when tracer dispersion is dominated by stirring, the equivalent variance is found to increase at a rate between the $2^{\text {nd }}$ power law (for a pure shearing flow regime) and the exponential law (for a pure stretching flow regime). The second method is based on the length of tracer contours. In the framework of equivalent radius, the two methods of inferring eddy diffusivity can be understood as two different averagings over the tracer patch. Over a shorter period of tracer dispersion the two methods give different eddy diffusivities and only over a longer time when tracer dispersion approaches the final stage of diffusion do they give a similar value of diffusivity. A new diagnostic quantity called stirring efficiency is introduced to indicate different flow regimes by measuring the efficiency of stirring against mixing. The new diagnostic quantity has the advantage that it can be calculated directly from the gradients of tracer distribution without needing to estimate strain rate or background diffusivity.
\end{abstract}




\section{Introduction}

It is well established that a scalar tracer released in a turbulent flow will be subject to stirring by the shear and mixing by the molecular diffusion. The stirring process is adiabatic with the tracer contours stretched and gradients sharpened. This creates small-scale features that eventually molecular diffusion acts upon, leading to irreversible mixing and smoother tracer gradients. Thus, the action of diffusion is enhanced by the shears of advective flow and one seeks an effective diffusivity that incorporates stirring-enhanced mixing. See Garrett (2006) for a review of stirring and mixing by turbulence in the ocean.

In the last decade or so, oceanographers have tried to infer eddy diffusivity by releasing inert tracers such as $S F_{6}$ into the ocean. Most tracer release experiments focus on estimating diapycnal mixing in a particular environment such as in the quiet main thermocline of the subtropical gyre (Ledwell et al. 1998), within the convection region in the Greenland Sea (Watson et al. 1999), over rough topography in the abyssal Brazil basin (Ledwell et al.2000), or associated with salt fingers in the main thermocline of the tropical Atlantic (Schmitt et al. 2005). Estimates of diapycnal diffusivity vary from $0.1 \mathrm{~cm}^{2} \mathrm{~s}^{-1}$ in the main thermocline to $10 \mathrm{~cm}^{2} \mathrm{~s}^{-1}$ or more in the presence of rough topography. Eddy diffusivity along isopycnals is rarely estimated from these experiments because it is almost impossible to recover all the tracer after the release. However, in the North Atlantic Tracer Release Experiment (NATRE) in 1992, the eddy isopycnal diffusivity was estimated to be about $1000 \mathrm{~m}^{2} \mathrm{~s}^{-1}$ (Ledwell et al. 1998). But, it is not clear how robust is such an estimate based on few observations with long time intervals between them. The purpose of this study is to examine in more detail the method of determining effective isopycnal eddy diffusivity from a point release tracer.

The dispersion of tracer has three distinct stages (Garrett 1983). Initially, tracer diffuses from a point into a Gaussian distribution. The area increases linearly with time at a rate proportional to small-scale (background) diffusivity. The second stage begins when the tracer patch reaches a length scale large enough for the stirring by shear flow to dominate the initial diffusion. Finally, when the length scale of the tracer patch is larger than that of the eddies, streaks are wrapped around and merged together, resulting in a more homogenized tracer patch. Thus, at a longer time, the ensemble averaged tracer diffuse in a Fickian manner with an eddy effective diffusivity. The three-stage tracer dispersion has been illustrated in a two-layer quasi-geostrophic vorticity model calibrated with the NATRE floats 
data (Sundermeyer and Price 1998).

Some characteristic of flows may be inferred from each stage of the dispersion: the background diffusivity from the initial stage, strain rate from the second stage and the eddy effective diffusivity over a longer time. However, the question is how can we identify which stage the tracer dispersion has reached? The most common measure is the rms distance from the centre of mass of tracer distribution. We will compare this with the other two measures: the area occupied by tracers (Joseph and Sender 1958) and the length of tracer contours (Nakamura 1996). These two measures are explained in details in section 2. Briefly here, the 'area' method measures the rate of increase of area enclosed by tracer contours. The 'contour length' method (called equivalent length) measures the length of interface available for background diffusion. Both are distinctly different from the rms distance to the center of mass method in their insensitivity to the shape of tracer distribution.

The equivalent length method is an elegant way of diagnosing stirring-enhanced mixing (Nakamura 1995). It is based on the fact that nondivergent flows are area (or volume) preserving and so advection alone cannot change the area (or volume) enclosed by tracer contours. However, the shear flow can deform tracer contours and the available interface for diffusion is elongated. Thus, the more complex the geometry of tracer distribution is, the more effective diffusion will be. Diagnosis of effective diffusivity using this method has been applied to quasi-steady chemical tracers in atmospheric models (Nakamura and Ma 1997; Allen and Nakamura 2001) and passive tracers in an idealised Southern Ocean model (Marshall et al.2006). In this study, we apply it to point release tracers and introduce two new diagnostic tools (the mean effective diffusivity and stirring efficiency) so the evolution of tracers can be quantified.

We use a global eddy-resolving model in two horizontal resolutions at $1 / 12^{\circ}$ and $1 / 4^{\circ}$ with tracer released at a location as close to the NATRE as possible. The details of the model experiments are given in section 3 and the evolutions of tracers are in section 4 . In section 5 and 6 , diagnoses using each method are illustrated and compared. The possible impact of diapycnal processes on the methods is discussed in section 7. There is a summary in section 8 . 


\section{Background}

\subsection{The equivalent radius variance}

Consider a simple 2-dimensional case where the evolution of tracer is controlled by diffusion,

$$
\frac{\partial C}{\partial t}=k_{x} \frac{\partial^{2} C}{\partial x^{2}}+k_{y} \frac{\partial^{2} C}{\partial y^{2}}
$$

where $C(x, y, t)$ is the tracer concentration and $k_{x, y}$ are the constant diffusivities in the $x$ and $y$ directions.

The solution for a tracer initially released at a point $(x, y)=(0,0)$ is a Gaussian distribution (e.g. Sanderson and Okubo 1986),

$$
C(x, y, t)=\frac{Q}{2 \pi \sigma_{x} \sigma_{y}} e^{-\left(\frac{x^{2}}{2 \sigma_{x}^{2}}+\frac{y^{2}}{2 \sigma_{y}^{2}}\right)},
$$

where $Q$ is the total tracer load and $\sigma_{x, y}^{2}=2 k_{x, y} t$ are the variances in the $x$ and $y$ directions. The quantitiy $\sigma_{x} \sigma_{y}$ is called the mean variance. The peak tracer concentration, $C_{\max }=\frac{Q}{2 \pi \sigma_{x} \sigma_{y}}$, decreases inversely with time and the contours of constant tracer concentration form a set of ellipses. The variance in the direction of the principal axes of the ellipses, $\sigma_{x, y}^{2}$, grows linearly in time at a rate twice the respective diffusivity.

In the ocean, there are spatially-varying flows and so a point-released tracer will no longer evolve into a simple Gaussian distribution with elliptical tracer contours. Instead, the action of shearing and stretching by differential advection causes tracer contours to be deformed into irregular shapes with steep gradients and fine filaments. In such situation, it may be preferrable to use some kind of variance without needing to specify any particular direction such as the directions of principle axes. One way to do this is to use the area enclosed by tracer contours (Joseph and Sender 1958, Okubo 1971), which we describe below.

For a given a tracer concentration value $c$, define an equivalent radius $\gamma_{e}(c)$ that satisfies $\pi \gamma_{e}^{2}=\mathcal{A}_{c}$, where $\mathcal{A}_{c}$ is the area enclosed by the tracer contour $C=c$. Thus, for any tracer distribution $C$ there is a corresponding radially symmetrical function $\hat{C}$ such that $\hat{C}\left(\gamma_{e}(c)\right)=c$. From this function, one obtains a tracer-weighted average of equivalent radius squared,

$$
\sigma_{e}^{2} \equiv \frac{\int \gamma_{e}^{2} \hat{C}\left(\gamma_{e}\right) d a}{\int \hat{C}\left(\gamma_{e}\right) d a}
$$


where the area element is $d a=2 \pi \gamma_{e} d \gamma_{e}$. We will call $\sigma_{e}^{2}$ the equivalent (radius) variance and $\sigma_{e}$ the equivalent deviation. A more intuitive interpretation of the equivalent variance is to observe that $\pi \sigma_{e}^{2}$ is simply the tracer-weighted average of the area enclosed by tracer contours.

In the case of a Gaussian distribution as in example (2), it is straightforward to show that the equivalent radius variance is twice the mean variance, $\sigma_{e}^{2}=2 \sigma_{x} \sigma_{y}$, and $\pi \sigma_{e}^{2}$ is equal to the amount of area enclosed by the contour of tracer concentration $c_{1}=C_{\text {max }} e^{-1}$. It is worth noting that the contour $C=c_{1}$ encompasses a tracer load of $Q\left(1-e^{-1}\right)$, which is about $63 \%$ of the total amount of tracer. This may be compared to the contour of tracer concentration $C_{\max } e^{-3}$, which encloses an area of $3 \pi \sigma_{e}^{2}$ that encompasses about $95 \%$ of total tracer load. We will use these facts later when comparing different methods of inferring diffusivity.

The equivalent variance, $\sigma_{e}^{2}$, is different from the conventional distance variance,

$$
\sigma_{r}^{2} \equiv \frac{\int r^{2} C(x, y) d a}{\int C(x, y) d a}
$$

where $r=\sqrt{x^{2}+y^{2}}$ is the distance to the center of mass and the area element is $d a=d x d y$. In the case of the simple diffusion example (2), the conventional distance variance is $\sigma_{r}^{2}=\sigma_{x}^{2}+\sigma_{y}^{2}$. In this case, it is clear that $\sigma_{e}^{2} \leqslant \sigma_{r}^{2}$. It can be shown that this inequality is always true for any distribution.

The common practice is to infer diffusivities from the growth rate of variance. In theory any variance can be used, but the inferred diffusivity will depend on the choice of variance for any distribution other than a symmetrical Gaussian distribution. All the variances mentioned so far except the equivalent variance strongly depend on the shape of the tracer distribution. For this reason, we would emphasize the use of equivalent variance and define an apparent diffusivity, as in Okubo (1971),

$$
\kappa_{a} \equiv \frac{1}{4} \frac{\partial \sigma_{e}^{2}}{\partial t}
$$

Thus, apparent diffusivity is a measure (up to a scale $4 \pi$ ) of how fast the tracer-weighted average of the area enclosed by tracer contours spreads, regardless of the geometrical shape of tracer distribution. The factor $1 / 4$ is such that $k_{a}$ gives the same diffusivity for a symmetric Gaussian distribution from a simple diffusion problem.

\subsection{The time evolution of variances}

How does a point-release tracer evolve in a turbulent flow ? Garrett (1983) described the dispersion 
of point release tracers as a three-stage process. Assume the ensemble averaged tracer distribution over many realisations is a Gaussian distribution and define the tracer area $\Gamma$ to be the area enclosed by the tracer contour with concentration value $c=$ peak value $\times e^{-1}$. Note that this way of defining $\Gamma$ uses the property of Gaussian distribution and so $\Gamma$ is a special case of the equivalent variance $\sigma_{e}^{2}$ because $\sigma_{e}^{2}$ can be applied to any distribution.

Initially, tracer diffuses from the point of release to a near symmetrical Gaussian distribution by small-scale diffusion with $\Gamma=4 \pi k t$, where $k$ is the background diffusivity. The diffusive process dominates until the length scale of tracer $L_{C}$ is comparable to the length scale of the flow $L_{U}$ and then stirring by shear flows begins to take effect. This is supposed to take place at the time when the advective time scale $L_{U} / U$ (where $U$ is the velocity scale) is shorter than the diffusive time scale $L_{C}^{2} / k$ (where $L_{C} \sim 2 \sqrt{k t}$, the radius of the circular area $4 \pi k t$ ). This implies a time scale $T_{a}=(4 \lambda)^{-1}$ and a tracer length scale $L_{C} \geq \sqrt{k / \lambda}$, where $\lambda=U / L_{U}$ is the scale of the strain rate. During the stirring-dominated stage, the distorted tracer patch is thought of as a deformed Gaussian distribution with $\Gamma(t)=\Gamma_{0} e^{\alpha \lambda\left(t-T_{a}\right)}$, where $\alpha$ is an $O(1)$ constant and $\Gamma_{0}=\left.\Gamma\right|_{t=T_{a}}$. This indicates that the tracer area $\Gamma$ increases exponentially in time at a rate proportional to the strain rate $\lambda$. Finally, when the length scale of the tracer is much larger than that of the flow, streaks of the tracer are wrapped around and eventually merged together by diffusion, resulting in a more homogenised tracer field. The tracer distribution at this final stage is nearly Gaussian with $\Gamma=4 \pi k_{h} t$, where $k_{h}$ is called the effective eddy diffusivity.

For some simple cases where the flow is steady, the tracer advection-diffusion equation can be solved explicitly (Okubo 1966). The simplest case is when there is no stretching, no shearing and only rotation, and so tracer is diffused by background diffusion. In the case of $u=u_{0}+\lambda y, v=0$ (pure shearing), the mean variance $\sigma_{x} \sigma_{y} \sim k \lambda t^{2}$ for large $t$ (Novokov 1958). In the case of $u=\eta x, v=$ $-\eta y$ (pure stretching), the mean variance $\sigma_{x} \sigma_{y} \sim \frac{k}{\eta} e^{2 \eta t}$ for large $t$ (Townsend 1951). The scalars $\lambda$ and $\eta$ are the constant shear rate and constant stretching rate, see Appendix B for definition. So, the mean variance increases in time as a power of 2 for pure shearing flows and exponentially for pure stretching flows. Therefore, Garrett's prediction of exponential growth of $\Gamma$ is at least consistent with the case for pure stretching flows. One might expect that in the ocean the variance growth rate is somewhere between the two flow regimes. In any case, during the second stage of tracer dispersion, the increase of variance will be faster than linear, so the apparent diffusivity will be time-dependent. 


\subsection{The transformed tracer equation}

The concept of equivalent radius is very useful in that apart from inferring the apparent diffusivity it can be used as a coordinate to simplify the tracer advection-diffusion equation. The equivalent radius coordinate has been applied to quasi-steady tracers for studying stirring and mixing in the ocean and atmosphere (Nakamura 1995, 1996, Shuckburgh and Haynes 2003, Marshall et al. 2006). Here, we apply it to a point-released tracer and compare to the apparent diffusivity in the previous section.

Consider the advection-diffusion equation of tracer,

$$
\frac{\partial C}{\partial t}+\nabla \cdot(\mathbf{u} C)=\nabla \cdot(k \nabla C)
$$

where $\mathbf{u}=(u, v)$ is the divergence-free velocity and $k$ is the constant background diffusivity. The derivation for rewriting (6) in equivalent radius coordinate can be found in the literatures. All our diagnosis uses the isopycnic layer thickness formulation (see the Appendix), but for the convenience of discussion the following equations omit the layer thickness.

In equivalent radius coordinates, the tracer equation (6) is transformed into a diffusion-only equation

$$
\frac{\partial \hat{C}\left(\gamma_{e}, t\right)}{\partial t}=\frac{1}{L_{0}} \frac{\partial}{\partial \gamma_{e}}\left(\kappa_{e} L_{0} \frac{\partial \hat{C}\left(\gamma_{e}, t\right)}{\partial \gamma_{e}}\right),
$$

where $\hat{C}\left(\gamma_{e}, t\right)$ is the radially symmetric function as before, $\kappa_{e}\left(\gamma_{e}, t\right)$ is the effective diffusivity,

$$
\kappa_{e}\left(\gamma_{e}, t\right)=k \frac{L_{e}^{2}\left(\gamma_{e}, t\right)}{L_{0}^{2}\left(\gamma_{e}, t\right)}
$$

$L_{e}\left(\gamma_{e}, t\right)$ is the equivalent length,

$$
L_{e}^{2}\left(\gamma_{e}(c), t\right)=\oint_{C=c} \frac{1}{|\nabla C|} d l \oint_{C=c}|\nabla C| d l
$$

and $L_{0}\left(\gamma_{e}, t\right)=2 \pi \gamma_{e}$ is the minimal length that a tracer contour can have for enclosing the same amount of area.

There is no explicit advective term in (7) since divergence-free flows are area-preserving and so $\hat{C}\left(\gamma_{e}\right)$ can only be changed by diffusion. That is to say, if there is no background diffusion $(k=0)$ then the value of tracer concentration enclosing a given amount of area will remain unchanged at all time and so the 1.h.s. of (7) will be zero. The crucial point of the transformed tracer equation is that if there are shears then the diffusion of tracer is much more effective than that given by the background 
diffusivity. The shear-enhanced diffusion is manifested in the effective diffusivity $\kappa_{e}$, which is the background diffusivity multiplied by a factor of $L_{e}^{2} / L_{0}^{2}$. The Cauchy-Schwartz inequality tells us that $L_{e}$ is always greater or equal to the actual length of the contour, $L=\oint_{C=c} d l$ (Shuckburgh and Haynes 2003). Clearly, $L \geq L_{0}$, the length of a tracer contour is always greater than the minimal length that a contour can have for the same enclosed area. Thus, the ratio $L_{e} / L_{0}\left(\geq L / L_{0}\right)$ measures how long a tracer contour is relative to the minimal length that a contour can have for the same enclosed area. The idea is that as a tracer contour is deformed by shear flow, the ratio $L_{e} / L_{0}$ increases, resulting in a longer interface for diffusion to operate and therefore a shear-enhanced diffusion.

The effective diffusivity $\kappa_{e}$ in (8) is an averaged diffusivity for each tracer contour, i.e., it can vary across tracer contours but not along the contours. The formulation of (8) implies that $\kappa_{e}$ can be calculated at any instant without prior knowledge of the history of a tracer. However, the disadvantage is that it is necessary to know the value of background diffusivity before one can obtain a realistic value of effective diffusivity.

To get around this problem, we propose an alternative way of calculating effective diffusivity which does not require the knowledge of background diffusivity or the gradients of tracer. Integrating (25) (from the Appendix) with respect to $c$ to give

$$
\int^{c} \frac{\partial \mathcal{A}}{\partial t} d c=-k L_{e}^{2} \frac{\partial c}{\partial \mathcal{A}}=-\kappa_{e} L_{0}^{2} \frac{\partial c}{\partial \mathcal{A}}
$$

where $\mathcal{A}(c)$ is the area enclosed by tracer concentration $c$. The first term is the total diffusive flux of tracer across a fixed contour $C=c$ and so (10) is a flux-gradient relationship. The effective diffusivity can be calculated from equating the total diffusive flux (the first term) to the third term in (10). Interestingly, the first equality in (10) implies that background diffusivity can also be calculated in a similar way.

At this point, it is worth comparing the effective diffusivity $\kappa_{e}$ to the apparent diffusivity $\kappa_{a}$ discussed in section 2.1. First of all, the effective diffusivity $\kappa_{e}$ is an averaged value for each tracer contour and so it can vary from contour to contour. This means that $\kappa_{e}$ has one degree of freedom in the horizontal space. In contrast, the apparent diffusivity $\kappa_{a}$ is an averaged quantity for the entire tracer distribution. Secondly, the effective diffusivity $\kappa_{e}$ in (8) gives an impression of its dependence on the length. However, if the effective diffusivity is expressed using the flux-gradient relationship (10), then $\kappa_{e}$ can be reformulated in the terms of the change of area enclosed by tracer contours. This bears 
some similarities to the apparent diffusivity $\kappa_{a}$ which is related to the rate of change of the mean area enclosed by tracer contours. Before we compare them further, we need to have some kind of averaged effective diffusivity for the entire tracer and not just for each tracer contour.

\subsection{The transformed tracer variance equation}

To do this, we make use of the transformed equation for tracer variance ${ }^{1}$. Multiply (7) by $\hat{C}\left(\gamma_{e}, t\right)$ and integrate over the tracer domain,

$$
\frac{\partial}{\partial t} \int \frac{1}{2} \hat{C}^{2}\left(\gamma_{e}, t\right) d a=-\int \kappa_{e}\left|\frac{\partial \hat{C}\left(\gamma_{e}, t\right)}{\partial \gamma_{e}}\right|^{2} d a
$$

where $d a=L_{0} d \gamma_{e}=2 \pi \gamma_{e} d \gamma_{e}$ is the area element. From (11), the mean effective diffusivity $\bar{\kappa}_{e}$ can be defined as,

$$
\frac{\partial}{\partial t} \int \frac{1}{2} \hat{C}^{2} d a \equiv-\bar{\kappa}_{e} \int\left|\frac{\partial \hat{C}}{\partial \gamma_{e}}\right|^{2} d a .
$$

Thus, the mean effective diffusivity $\bar{\kappa}_{e}$ is the ratio between the rate of change of total tracer variance $\int \frac{1}{2} \hat{C}^{2} d a$ and the total tracer gradients squared in equivalent radius coordinate. Alternatively, equate (11) and (12) to obtain

$$
\bar{\kappa}_{e}=\frac{\int \kappa_{e}\left|\frac{\partial \hat{C}\left(\gamma_{e}, t\right)}{\partial \gamma_{e}}\right|^{2} d a}{\int\left|\frac{\partial \hat{C}}{\partial \gamma_{e}}\right|^{2} d a} .
$$

Thus, the mean effective diffusivity $\bar{\kappa}_{e}$ is the average of effective diffusivity $\kappa_{e}\left(\gamma_{e}(c)\right)$ weighted by the squared tracer gradient. The weighted average means that more weight is placed toward the centre of a tracer patch where the tracer contours are more compact. This interpretation of $\bar{\kappa}_{e}$ will help us to understand the comparison with the apparent diffusivity.

It is also worth comparing the transformed tracer variance equation (11) with the conventional tracer variance equation,

$$
\frac{\partial}{\partial t} \int \frac{1}{2} C^{2}(x, y, t) d a=-k \int|\nabla C|^{2} d a
$$

where $d a=d x d y$. By comparing (14) with (12), it can be seen that the effect of shear on tracers is embedded in the tracer gradients when using $(x, y)$ coordinates whereas it is automatically included in the mean effective diffusivity when using equivalent radius coordinates.

\footnotetext{
${ }^{1}$ The term variance is not to be confused with the variance w.r.t. distribution, such as $\sigma_{r}^{2}$.
} 
Since the total variance is the same regardless of the coordinates, the 1.h.s. of (11) and (14) are identical. Take the ratio to define the 'stirring efficiency' as

$$
\zeta \equiv \frac{\int|\nabla C|^{2} d a}{\int\left|\frac{\partial \hat{C}}{\partial \gamma_{e}}\right|^{2} d a} .
$$

By definition, stirring efficiency measures how much steeper tracer gradients are relative to the gradients that the tracer would have if it had not been distorted by shear flows. Thus, it is an indicator of how efficient differential advection is sharpening the tracer gradients against the background diffusion which acts to smooth the tracer gradient. It is not difficult to see that in fact $\zeta=\bar{\kappa}_{e} / k$ and so $\zeta$ also tells us how much efficient diffusion has become as a result of stirring. However, the real advantage of defining the stirring efficiency as we did in (15) is that it simply uses the degree of tracer distortion to tell us something about stirring vs. mixing without needing to calculate strain rate and small-scale diffusivity separately. This way of using tracers to obtain flow information is particularly useful for the real ocean where strain rate and background diffusivities are difficult to measure.

\subsection{Comparing effective and apparent diffusivities}

We are now ready to compare the effective and apparent diffusivities. Using the transformed tracer equation (7), we can express the apparent diffusivity in the following way. First, we rewrite the time derivative of the numerator of the equivalent variance (3),

$$
\frac{\partial}{\partial t} \int \gamma_{e}^{2} \hat{C} d a=\int \gamma_{e}^{2} \frac{\partial \hat{C}}{\partial t} d a=\int \gamma_{e}^{2} \frac{1}{L_{0}} \frac{\partial}{\partial \gamma_{e}}\left(\kappa_{e} L_{0} \frac{\partial \hat{C}}{\partial \gamma_{e}}\right) d a=4 \pi \int \frac{\partial}{\partial \gamma_{e}}\left(\kappa_{e} \gamma_{e}^{2}\right) \hat{C} d \gamma_{e}
$$

The last equality uses integration by parts twice. Use $\mathcal{A}=\pi \gamma_{e}^{2}$ (the area enclosed by the contour $C=c)$ as a variable to rewrite the r.h.s. of (16),

$$
\frac{\partial}{\partial t} \int \gamma_{e}^{2} \hat{C} d a=4 \int \frac{\partial}{\partial \mathcal{A}}\left(\kappa_{e} \mathcal{A}\right) \hat{C} d a
$$

Since the total amount of tracer is constant, the apparent diffusivity from (5) can be written as

$$
\kappa_{a}=\frac{\int \frac{\partial}{\partial \mathcal{A}}\left(\kappa_{e} \mathcal{A}\right) \hat{C} d a}{\int \hat{C} d a}
$$

In this way, the apparent diffusivity is interpreted as the tracer-weighted average of $\frac{\partial}{\partial \mathcal{A}}\left(\kappa_{e} \mathcal{A}\right)$. The unusual outcome is that the quantity $\frac{\partial}{\partial \mathcal{A}}\left(\kappa_{e} \mathcal{A}\right)$ takes into account the across-contour variation of effective diffusivity $\kappa_{e}$. 
So, (13) and (18) together tells us that the mean effective diffusivity $\bar{\kappa}_{e}$ and the apparent diffusivity $\kappa_{a}$ can be interpreted as two different ways of averaging $\kappa_{e}$. There is no particular reason to believe why one should be better than the other. The diffusivities $\kappa_{a}$ and $\bar{\kappa}_{e}$ can be calculated anytime during the tracer dispersion. During the initial background diffusion dominant stage, the two diffusivities should give similar values since there is not much tracer distortion. During the stirring-dominant stage, diffusivities $\kappa_{a}$ and $\bar{\kappa}_{e}$ will deviate from each other since each averaging procedure will bias different aspect of the tracer distribution. At much longer times when tracer contours are merged and the tracer distribution is close to Gaussian, $\kappa_{a}$ and $\bar{\kappa}_{e}$ should converge a similar value again since now the ratio $L_{e} / L_{0}$ (hence $\kappa_{e}$ ) is nearly constant. So, over the longer time, it is expected all three eddy diffusivities discussed so far will be similar, $k_{h} \sim \kappa_{a} \sim \bar{\kappa}_{e}$.

\section{Model}

The model we use is a global ocean model, the Ocean Circulation and Climate Advanced Model (OCCAM) (Coward and de Cuevas, 2005). Here we only describe the part of model setup which is relevant to the passive tracer experiment. Other model details may be found in Lee et al. (2007). The OCCAM model has a suite of runs with three different horizontal resolutions at $1^{\circ}, 1 / 4^{\circ}$ and $1 / 12^{\circ}$. All runs have the same 66 vertical levels and the same six-hourly atmospheric fields (from NCEP/NCAR) for calculating surface fluxes. Note that the applied surface fluxes depend on the surface ocean temperature and so may be different in different runs. For our study, we use the runs at $1 / 4^{\circ}$ and $1 / 12^{\circ}$ resolutions. The horizontal advection of tracers uses the modified split QUICK (MSQ) scheme. This involves a 4th-order accurate advection scheme together with a velocity dependent biharmonic diffusion. There is no explicit horizontal diffusion. The vertical advection uses simple second order centre differences with an explicit diffusivity of $0.1 \mathrm{~cm}^{2} \mathrm{~s}^{-1}$. In the $1 / 4^{\circ}$ run, there is also Gent and McWilliams parameterization and isopycnic diffusion with both thickness diffusivity and isopycnal diffusivity set to be $50 \mathrm{~m}^{2} \mathrm{~s}^{-1}$.

\subsection{Tracer releases}

To better understand how the diffusivity metrics described in Section 2 relate to the real ocean, we perform a series of numerical tracer experiments designed to be as similar as possible to the the 
North Atlantic Tracer Release Experiment. Although we cannot replicate the exact conditions of NATRE, the general conditions of an open ocean pycnocline tracer release is a useful setting for better understanding the different measures of diffusivity. The NATRE release site is located in the southeastern part of the subtropical gyre in the North Atlantic at a depth of about 300 m over a region about $25 \mathrm{~km}$ by $25 \mathrm{~km}$ in the horizontal. The tracer in the model is injected into nine grid cells in the $1 / 12^{\circ}$ run with $(3,3,1)$ in $(x, y, z)$ direction and into one grid cell in the $1 / 4^{\circ}$ run. The centre of each tracer patch (the triangle marker in Fig. 1) is as close to that in the NATRE as model grids allow. Despite this, there are still considerable differences between the model and observational releases. At $300 \mathrm{~m}$, the model vertical grid spacing is about $34 \mathrm{~m}$, much greater than the $2 \mathrm{~m}$ vertical spread in the NATRE. The tracer in the models is set to be uniformly 1 throughout the initialization grid cells. In the NATRE, the tracer is released along cruise tracks and so they are streaky and discontinuous.

When the tracer varies over a length scale, $L_{C}=\delta \Delta x$, the biharmonic diffusion associated with the horizontal advection scheme implies a diffusivity $k^{\prime}=1 / 16 U(\Delta x)^{3} /\left(L_{C}^{2}\right)$ (where $\delta$ is a scalar, $U$ is the velocity scale and $\Delta x$ is the grid spacing) (Webb et al. 1998, Lee et al. 2002). The biharmonic diffusion becomes larger than the advection when $k^{\prime} / L_{c}^{2} \geq U / L_{U}$, which implies $\delta \leq 1 / 2\left(L_{U} / \Delta x\right)^{1 / 4}$. Let $U \sim 4 \mathrm{~cm} \mathrm{~s}^{-1}$ and the strain rate $\lambda \sim 10^{-6} \mathrm{~s}^{-1}$ (these are the averaged values over the tracer patch in the $1 / 12^{\circ}$ model run), then the length scale of the flow is $L_{U}=U / \lambda \sim 40 \mathrm{~km}$ and the scale of tracer is $L_{C} \sim 1 / \sqrt{2} \Delta x$. So, the scale-selected biharmonic diffusion ensures that tracer filaments are marginally resolved at the model grid scale.

The models start from 1985 (see Coward and de Cuevas 2005 for model start-up). Tracer are integrated on-line, but because of the limitation of disk space and computational resources we can only run one tracer at one time. The model tracers are not released at the same year as in the NATRE, but they were released at the same month whenever possible. For the $1 / 4^{\circ}$ run, a test run of tracer was started in May 1993 and run for 360 days (called EXP1/4test). The tracer was reinitialized in May 1994 and run until 2002 (called EXP1/4). For the 1/12 ${ }^{\circ}$ run, a test run was started in August 1994 and run for 260 days (called EXP1/12 $2_{\text {test }}$ ). The tracer was reinitialised in May 1995 and run until 2000 (called EXP1/12). The two test runs allow us to assess the sensitivity to the release time. Our main results will be focused on the first three years of two main runs EXP1/4 and EXP1/12. For convenience, we use elapsed days since the release without referring to specific months and years.

\subsection{Method of binning}


The $z$-level data is binned into 10 density layers defined by potential density $\sigma_{.3}$ referenced to $300 \mathrm{~m}$. The potential density classes are 27.1, 27.3, 27.5, 27.7, 27.9, 28.1, 28.3, 28.5, 28.7, 28.9 (in units of $\mathrm{kg} \mathrm{m}^{-3}$ ). We use the same binning procedure as in Lee et al. (2007), so ensuring properties (mass, and tracer substance) are conserved. On the density layer, the tracer concentration is defined to be the layer-thickness weighted mean $\widehat{\tau}(x, y, t)=\overline{\tau h} / \bar{h}$, where the overbar is the vertical integral over the target density layer. Note that any binning procedure will inevitably create averaged tracer values, so tracer on a density layer might appear to be more mixed than on a z-level. However, we tested many different choice of density bins and the results do not change qualitatively. For the rest of paper, we will use the notation $\tau$ rather than $\widehat{\tau}$ for convenience. The target density surface in the NATRE is 28.05. In the model, the density layer that contains most of the initial tracer substance is the density layer 27.9, which will be our model target density layer.

Once properties are binned into isopycnic layers, we perform a second binning procedure by binning properties according to the tracer value on the target density layer. The tracer-based binnings allow us to calculate quantities such as the area enclosed by tracer contours on the target density layer. As the tracer concentration is diluted in time, we need to have tracer bins that also vary with time, unlike the density bins which are time independent. We choose the values for tracer bins according to an exponential function $\left\{C_{\max }(t) \exp (3(n / N-1)), n=1, N\right\}$, where $C_{\max }(t)$ is the maximum value of $C(x, y, t)$ in the target density layer. The number of tracer bins is $N=20$ and $N=40$ for the $1 / 4^{\circ}$ and $1 / 12^{\circ}$ runs, respectively. The smaller number of bins for the $1 / 4^{\circ}$ run is to avoid too much noise caused by the tracer occupying very few grid cells initially. Two additional bins -1 and 0 were added to account for negative tracer values. The negative value tracer typically takes up about $0.2 \%$ of total tracer substance.

In section 2, we have assumed no diapycnal flow. However, models do have diapycnic flows and so the total tracer substance in the density layer will not be exactly conserved. Between 5-day means, tracer substance in the target density layer changes by no more than $0.3 \%$ in both $1 / 4^{\circ}$ and $1 / 12^{\circ}$ runs. By the end of three years about $60 \%$ and $70 \%$ of the total tracer substance remains in the target density layer for the $1 / 12^{\circ}$ run and $1 / 4^{\circ}$ run, respectively. We will discuss how this might affect diagnostic methods in section 6.4. 


\section{Evolution of tracer}

As in the NATRE, the tracers in the model were released in a region where eddy kinetic energy is relatively low compared to that in the vicinity of the Gulf Stream. Figure 1 (shading) shows annual mean eddy kinetic energy at $314 \mathrm{~m}$ (model level 25) for year 1995 from the $1 / 12^{\circ}$ run. The superimposed contours show the year 1995 annual mean pressure field at $314 \mathrm{~m}$, indicating the largescale circulation of the subtropical gyre. However, flows are by no means quiescent. At the time of release, the pressure at $314 \mathrm{~m}$ in the $1 / 12^{\circ}$ run is filled with small-scale turbulence (Figs. 2ab, the relative size of box is drawn in Fig. 1). The eddy-rich structure has a great impact on the initial spreading of tracer. In EXP1/12 $2_{\text {test }}$, the release site was near the southwestern edge of a cyclonic eddy (Fig. 2a). At day 180, the tracer becomes elongated in the east-west direction and the centre of mass is to the southwest direction following the tailend of the cyclonic eddy (Fig. 2c). In contrast, the release site in EXP1/12 was near the southwestern edge of an anticyclonic eddy (Fig. 2b). As a result of following the anticyclonic eddy, the centre of mass at day 180 is to the northeast direction (Fig. 2d), opposite to that in the test run EXP1/12 $2_{\text {test }}$.

On the other hand, the tracers in the $1 / 4^{\circ}$ run are not as sensitive to the time of release as in the $1 / 12^{\circ}$ run. This is because there is no small-scale eddy field in the $1 / 4^{\circ}$ run, as shown by the pressure fields (Figs. $3 \mathrm{ab}$ ). For both EXP1/4 and EXP1/4test, the centre of mass at day 180 is to the southwest and the spatial pattern remains near-circular with only slight deformation (Figs. 3cd).

In the NATRE, the centre of mass moves to the southwest after 6 months, similar to EXP1/12 ${ }_{\text {test }}$. However, as we have demonstrated this clearly depends on the flow field at the time of release. Since the model does not reproduce exactly the same flow in real time, we do not expect the model tracer to have the same distribution as in the NATRE. Our focus is on the characteristics of the spatial pattern. The tracer in the $1 / 12^{\circ}$ run is similar to the NATRE in that after 180 days it has a rich spatial structure with filaments and pinched tracer contours, which are completely absent from the $1 / 4^{\circ}$ run.

The continuous straining by the flow in the $1 / 12^{\circ}$ run eventually breaks up the tracer into four or five patches (Fig. 4, upper panels). After 365 days, it spreads over $10^{\circ}$ in the east-west direction and $7^{\circ}$ in the north-south direction. By the end of 2 years, the tracer has spread over a considerably wider region (the upper right panel shows part of whole region covered by tracer). In comparison, tracer in the $1 / 4^{\circ}$ run is slow to develop (Fig. 5, upper panels). It begins to stretch in the east-west 
direction after 180 days and becomes more distorted at the end of 2 years. However, it remains as a self-contained entity without fine filaments or breakups. Such differences between the two model tracers are due to the different strain rate of the flows, which will be discussed further in later sections. But first, we present the diagnosis of effective eddy diffusivity.

\section{The apparent diffusivity $\kappa_{a}$}

\subsection{The equivalent radius variance}

Figure 6 shows the time series of the equivalent variance, $\sigma_{e}^{2}$, and the conventional distance variance, $\sigma_{r}^{2}$. To take a closer examination of $\sigma_{e}^{2}$, we plot the time series in log-log scale (Fig. 7, left panels). The initial linear growth period of $\sigma_{e}^{2}$ seems to be reproduced well by $\sigma_{e}^{2}(0)+4 k t$ (thin solid lines), where $\sigma_{e}^{2}(0)$ is the variance at the time of release, and the background diffusivity $k$ is set to be 15 and $60 \mathrm{~m}^{2} \mathrm{~s}^{-1}$ for the $1 / 12^{\circ}$ and $1 / 4^{\circ}$ run, respectively. For the $1 / 12^{\circ}$ run, there is no explicit horizontal diffusion and so the background diffusivity arises from the implicit biharmonic diffusion in the horizontal advection scheme. For the $1 / 4^{\circ}$ run, the larger background diffusivity is due to the additional explicit diffusivity of $50 \mathrm{~m}^{2} \mathrm{~s}^{-1}$ from the isopycnal diffusion. So the mean numerical diffusivity is estimated to be of order $10-15 \mathrm{~m}^{2} \mathrm{~s}^{-1}$. These values are large compared to the estimate of the NATRE, where the diffusivity is about $2 \mathrm{~m}^{2} \mathrm{~s}^{-1}$ for the scale between 1 and $10 \mathrm{~km}$.

During the stirring-dominant stage, we compare the growth of $\sigma_{e}^{2}$ with two curves: one is $\lambda k t^{2}$, corresponding to the pure-shearing flow regime and the other is $\left.\sigma_{e}^{2}\right|_{t=T} e^{\alpha \eta(t-T)}$, corresponding to the pure-stretching flow regime. We set $T=70$ and 360 days for EXP1/12 and EXP1/4, respectively, and strain rate $\lambda=u_{y}+v_{x}$ and stretching rate $\eta=u_{x}-v_{y}$ to be the yearly-averaged values for the corresponding period $\left(1^{\text {st }}\right.$ year for EXP1/12 and $2^{\text {nd }}$ year for EXP1/4, see Appendix B). Although the parameter $\alpha$ is predicted to be order 1 (Garrett 1983), here the value 0.2 gives us a closer fit to $\sigma_{e}^{2}$ for both $1 / 12^{\circ}$ and $1 / 4^{\circ}$ runs. We see that in the $1 / 12^{\circ}$ run the rate of increase lies between the $t^{2}$ law (for a pure-shearing flow regime) and the exponential law (for a pure-stretching regime). Using the observed dye data Okubo (1971) suggested a $t^{2.3}$ law while from the NATRE tracer Ledwell et al. (1998) diagnosed a law close to $t^{2}$. It is less clear for the $1 / 4^{\circ}$ run because the stirring-dominant stage is not long enough yet to separate $t^{2}$ from exponential growth. 
If we assume that variance is controlled by the pure-shearing flow regime, $\sigma_{e}^{2} \sim \lambda k t^{2}$, then $\sigma_{e} \sim$ $\sqrt{\lambda k}$. The quantity $\frac{\partial \sigma_{e}}{\partial t} \sim \sqrt{\lambda k}$ is called diffusion velocity (Okubo 1968). This means that the rate of increase of equivalent deviation $\sigma_{e}$ is controlled by the combined effects of shearing and background diffusion. To verify this scaling using the two model runs, we need to choose the period from each model run when the rate of variance increase is closest to the power of 2 law (the first year for EXP1/12 and the second year for EXP1/4). From the diagnosed $\lambda$ and $k$ over these periods, we estimate the diffusion velocity $\sqrt{\lambda k}$ to be about $0.42 \mathrm{~cm} \mathrm{~s}^{-1}$ and $0.27 \mathrm{~cm} \mathrm{~s}^{-1}$ for EXP1/12 and EXP1/4, respectively. These diffusion velocities are comparable to the estimate of $1 \mathrm{~cm} \mathrm{~s}^{-1}$ by Joseph and Sender (1958). The time series of equivalent deviation $\sigma_{e}$ (Fig. 8) shows that the increase of $\sigma_{e}$ is about twice in EXP1/12 than in EXP1/4, which is close to the ratio of diffusion velocity $0.42 / 0.27 \sim 1.6$.

Garrett (1983) estimated that the time scale for tracer dispersion to reach the final stage of diffusion is about 1 year. His estimate is based on the exponential growth rate during the stirring-dominant stage and $k_{h}=1000 \mathrm{~m}^{2} \mathrm{~s}^{-1}$ for eddy diffusivity, $k=10^{-2} \mathrm{~m}^{2} \mathrm{~s}^{-1}$ for background diffusivity, $10^{-6}$ $\mathrm{s}^{-1}$ for strain rate and $\alpha=0.5$. For our model, if we take background diffusivity $k=10 \mathrm{~m}^{2} \mathrm{~s}^{-1}$ and $\alpha=0.2$, then we also obtain an estimated time scale about 365 days. If we instead use the $2^{\text {nd }}$ power law, then $4 k_{h} t=\lambda k t^{2}$ implies $t \sim 4 k_{h} /(\lambda k) \sim 12$ years. However, the growth rate of variance in the model is slower than exponential but faster than $2^{\text {nd }}$ power and so it is likely that the time scale for approaching final diffusion is order of 5-6 years. In any case, it can be seen from Fig. 7 that $\sigma_{e}^{2}$ has not reached the linearly increasing stage after three years.

So, we cannot estimate the eddy diffusivity $k_{h}$ from the growth of equivalent variance $\sigma_{e}^{2}$. However, we can still calculate the apparent diffusivity, $\kappa_{a}=\frac{1}{4} \frac{\partial \sigma_{e}^{2}}{\partial t}$, between two 5-day means (solid line in Fig. 9). Since the rate of growth of $\sigma_{e}^{2}$ after the initial period is faster than linear, the apparent diffusivity must be time dependent. During the first 500 days of EXP1/12, $\kappa_{a}$ increases to 1500 $\mathrm{m}^{2} \mathrm{~s}^{-1}$ and then fluctuates between 1000 and $1800 \mathrm{~m}^{2} \mathrm{~s}^{-1}$. Since the tracer in $1 / 12^{\circ}$ and $1 / 4^{\circ}$ models evolves at different time scales, one way to compare the corresponding apparent diffusivity is to see how they vary as functions of equivalent deviation, $\sigma_{e}$. If we assume the pure-shearing flow regime $\left(\sigma_{e}^{2} \sim \lambda k t^{2}\right)$, then $\kappa_{a} \sim \lambda k t \sim \sqrt{\lambda k} \sigma_{e}-$ the diffusion velocity appears again. Figure 10 shows that when $\sigma_{e}=250 \mathrm{~km}$ (corresponding to 450 days in EXP1/12 and 900 days in EXP1/4), the $\kappa_{a}$ is about 350 and $180 \mathrm{~m}^{2} \mathrm{~s}^{-1}$ for each run. The ratio of the diffusivities is again closer to that given by 
the diffusion velocity. This suggests it may not be unreasonable to scale $\kappa_{a}$ with a length scale, say, $\sigma_{e}$ and the diffusion velocity $\sqrt{\lambda k}$.

\subsection{Comparison with $\sigma_{r}^{2}$ and $\Gamma$}

Figure 6 shows that the equivalent variance evolves differently from the conventional variance. During the first 300 days, $\sigma_{r}^{2}$ increases more rapidly than $\sigma_{e}^{2}$ and afterwards more slowly than $\sigma_{e}^{2}$. A seemingly robust feature is $\sigma_{r}^{2} \geq \sigma_{e}^{2}$.

The time series in log-log scale shows that the conventional variance $\sigma_{r}^{2}$ in EXP1/12 (Fig. 7, upper

right panel) seems to approach a linear growth after about one year (Note that in the $1 / 4^{\circ}$ run, $\sigma_{r}^{2}$ has not reached the final diffusion stage yet). As predicted by Garrett (1983), $\sigma_{r}^{2}$ (in the $1 / 12^{\circ}$ run) indeed approaches the final diffusion stage earlier than $\sigma_{e}^{2}$ (or his $\Gamma$ ). This means while the distance to the centre of mass is not growing as fast as during the stirring dominant stage, the area covered by tracer continues the faster than linear growth, implying that the tracers contours are merged to 'fill in' the gaps between contours. This 'filling in' can also be seen from the time series of areas inside tracer contours containing $65 \%$ and $95 \%$ tracer load (Fig. 6). We see that $\pi \sigma_{r}^{2}$ is close to the $95 \%$ curve near the end of three years, but much greater than $95 \%$ over the first year because of the much more irregular tracer distribution during stirring-dominant stage.

From the linear growth of $\sigma_{r}^{2}$, we estimate the eddy diffusivity according to $\pi \sigma_{r}^{2}=12 \pi k_{h} t$. Note that here we use $12 \pi k_{h} t$ rather than $4 \pi k_{h} t$ because $\pi \sigma_{r}^{2}$ is closer to the area with $95 \%$ tracer load, and (see section 2.1) for a Guassian distribution the area containing $95 \%$ tracer load is $\sim 12 \pi k_{h} t$. This implies $k_{h} \sim 1000 \mathrm{~m}^{2} \mathrm{~s}^{-1}$. On the other hand, the equivalent variance $\sigma_{e}^{2}$ is very close to the $65 \%$ curve. Note that neither the $65 \%$ nor the $95 \%$ curve approaches the final diffusion stage (Fig. 6). Assuming $\sigma_{r}^{2}$ reached the final diffusion at year 1 , then the radius of the tracer patch would be $2 \sqrt{3 k_{h} t} \sim 660 \mathrm{~km}$. This suggests $\sigma_{e}$ needs to be about $660 \mathrm{~km}$ before tracer dispersion enters the final diffusion stage. If $\sigma_{e}$ follows the $2^{\text {nd }}$ power law, then this would take about 5 or 6 years.

For completeness, we also calculate $\Gamma$ (Fig. 6) using the simple relationship $\int C^{2} d a=\frac{Q^{2}}{2 \Gamma}$ (Garrett, 1983). It shows that the time evolution of $\Gamma$ is very similar to those of the equivalent radius variance, $\pi \sigma_{e}^{2}$, and also to the area enclosing $65 \%$ tracer load. We mentioned in section 2.1 that if the tracer distribution is Gaussian, then $\pi \sigma_{e}^{2}$ is equal to the area containing $65 \%$ tracer load and $\Gamma$ is equivalent 
to $\pi \sigma_{e}^{2}$. Although these two integral properties seem to hold for our tracer, it does not necessarily imply that the tracer distribution is Gaussian for a single realisation. In fact, we found $c \frac{\partial \mathcal{A}}{\partial c}$ is not spatially constant as it would be for a Gaussian distribution (not shown).

\section{The Effective diffusivity $\kappa_{e}$}

\subsection{Examples}

Here, we show examples of $\kappa_{e}$ as a function of tracer contours at a given 5-day mean. The effective diffusivity $\kappa_{e}$ can be evaluated from (8), but we need to first estimate background diffusivity. We could have used the background diffusivity $k$ obtained from the initial linear growth of the equivalent variance $\sigma_{e}^{2}$ in the previous section, but for an independent estimate we will use the tracer variance equation (14). The three years time series of the background diffusivity is shown in Fig. 11 (the thick dashed lines). The large spikes at the beginning of the $1 / 4^{\circ}$ run are due to the steep gradients resulting from the tracer taking up very few grid points initially. The 3-yr average value is about $19.6 \pm 4.8$ $\mathrm{m}^{2} \mathrm{~s}^{-1}$ and $90 \pm 22 \mathrm{~m}^{2} \mathrm{~s}^{-1}$ for EXP1/12 and EXP1/4, respectively (where \pm means one standard deviation). Thus, the background diffusivity estimated previously from the initial growth rate of $\sigma_{e}^{2}$ lies near the lower bound of the present estimate. As we expected, the background diffusivities for the two test runs (the thin dashed lines) do not differ significantly from those in the main runs.

We also calculate how the background diffusivity varies as a function of tracer contours using the first equality in (10). We choose one example from EXP1/12 at day 360 and one example from EXP1/4 at day 730 and plot them as functions of the equivalent radius (the dashed lines in Fig. 12). The background diffusivity does not seem to vary much across tracer contours.

We now present the equivalent length, $L_{e}$, the minimum length, $L_{0}$, and the actual length, $L$, as functions of tracer contours from EXP1/12 at day 360 (Fig. 13). Note that in calculating lengths all the line integrals are replaced by area integrals using the identity (26) to avoid integrating along curves. The equivalent length $L_{e}$ is longer than the actual length $L$ as expected from the CauchySchwartz inequality. The equivalent length $L_{e}$ is closer to the minimum length $L_{0}$ near the centre of the patch and increases to at least 10 times longer towards the edge of the tracer patch. This is due to the fact that the tracer contours at the outer edge of the tracer patch cover wider areas and so allow 
more stirring by the shear flow. This may be explained by the following scaling. The equivalent length may be scaled as $L_{e} \sim A \sqrt{\lambda / k}$, where $A$ is the size of the mixing region with strain rate $\lambda$ and $\sqrt{k / \lambda}$ is the width scale of filaments (Shuckburgh and Haynes 2003). Let $A \sim \gamma_{e}^{2}$, then $L_{e} \sim \gamma_{e}^{2} \sqrt{\lambda / k}$, suggesting $L_{e}$ varies as the square of the equivalent radius $\gamma_{e}$. So, $L_{e} / L_{0} \sim \gamma_{e} \sqrt{\lambda / k}$. This means that a larger area enclosed by contours (or, equivalently, a longer tracer contour) corresponds to a higher ratio of equivalent length to minimal length. The example in EXP1/12 shows that $L_{e}$ indeed increases faster than $\gamma_{e}$ but slightly slower than $\gamma_{e}^{2}$ (Fig. 13). Similarly, $L_{e} / L_{0}$ increases with $\gamma_{e}$.

In Fig. 12 (the dotted lines), the effective diffusivity $\kappa_{e}=k L_{e}^{2} / L_{0}^{2}$ is shown as a function of equivalent radius. For the background diffusivity, we should use the spatial-mean value at the specified day rather than the time-mean value. That is, $k=15.7$ and $62 \mathrm{~m}^{2} \mathrm{~s}^{-1}$ for the $1 / 12^{\circ}$ run at day 360 and for the $1 / 4^{\circ}$ at day 730 , respectively. The larger $\kappa_{e}$ towards the edge of the patch reflects the fact that there is a larger ratio $L_{e} / L_{0}$ as explained in Fig. 13. From the scaling $L_{e}^{2} / L_{0}^{2} \sim \gamma_{e}^{2} \lambda / k$, we can scale $\kappa_{e} \sim \lambda \gamma_{e}^{2}$. So, at a given $\gamma_{e}$, the effective diffusivity $\kappa_{e}$ is scaled by the straining rate. If we compare $\kappa_{e}$ in the $1 / 12^{\circ}$ and $1 / 4^{\circ}$ at the same equivalent radius $\gamma_{e}$, say, at $200 \mathrm{~km}$, then the ratio of $\kappa_{e}$ is about 3 although the ratio of strain rate gives about 6-10 (Fig. 12).

The alternative way of calculating $\kappa_{e}$ using the flux-gradient relationship (10) is shown by the solid lines in Fig. 12. They show that $\kappa_{e}$ from (10) is similar to that from (8). Since this method does not depend on the background diffusivity, the similarity between the two reassures us that the spatialmean choice of background diffusivity for (8) is fairly good. However, such similarity will not hold if we were to use the time-mean background diffusivity for (8), especially for the $1 / 4^{\circ}$ run where the background diffusivity varies considerably with time.

The effective diffusivity can be mapped onto the horizontal plane using the value of tracer at each point in space (lower panels in Figs. 4 and 5). The mapping is purely for visualization: recall that $\kappa_{e}$ does not vary along the contour of constant tracer concentration. Within each time frame, it can be seen that more deformed tracer contours correspond to larger effective diffusivity. Looking at the tracer patch as a whole, the overall effective diffusivity increases in time, reflecting more distortion of tracer as time increases. It is worth noting that the effective diffusivity in the $1 / 4^{\circ}$ run at day 730 has a similar order of magnitude to the $1 / 12^{\circ}$ run at day 180 . Since the background diffusivity in the $1 / 4^{\circ}$ run is about four times larger than that in $1 / 12^{\circ}$ run, this means that the $L_{e q}^{2} / L_{o}^{2}$ is much smaller in the $1 / 4^{\circ}$ run than that in the $1 / 12^{\circ}$ run. One can see that the tracer in the $1 / 12^{\circ}$ run at day 
180 has steeper gradients with fine filaments. Thus, the same value of effective diffusivity does not necessarily imply the same effect of eddy stirring on the tracer. Different parts of the ocean may have different background diffusivities at different times, thus one needs to be cautious about how to quantify the effect of eddies on the dispersion of tracer. In this sense, the 'stirring efficiency' $\zeta$ in (15) may turn out to be a useful quantity. We discuss this after we consider the time series of mean effective diffusivity.

\subsection{The mean effective diffusivity $\bar{\kappa}_{e}$}

The time series of the mean effective diffusivity $\bar{\kappa}_{e}$ using (13) is shown in Fig. 11 (the thick solid lines). The effective eddy diffusivity increases in time as the tracer evolves into a more complex distribution. The diffusivity in EXP1/12 starts at about $20 \mathrm{~m}^{2} \mathrm{~s}^{-1}$ initially and increases to about 254 $\mathrm{m}^{2} \mathrm{~s}^{-1}$ at day 180. After this, the large fluctuations continue and reach $500 \mathrm{~m}^{2} \mathrm{~s}^{-1}$ at day 500 and $850 \mathrm{~m}^{2} \mathrm{~s}^{-1}$ at day 700 or 800 . The increase carries on until the end of 3 years. EXP1/4 shows a sharp transition at first 40 days, which is due to steep gradients caused by the initial tracer occupying only one grid cell. After the initial period, there is a gradual increase from $50 \mathrm{~m}^{2} \mathrm{~s}^{-1}$ to about $250 \mathrm{~m}^{2} \mathrm{~s}^{-1}$ at day 600. The time series of $\bar{\kappa}_{e}$ from the two test runs EXP1/12 $2_{\text {test }}$ and EXP1/4 test are also shown (the thin solid lines). They exhibit slightly different patterns from those in the main runs. In particular, $\bar{\kappa}_{e}$ in EXP1/4test is consistently lower than in EXP1/4. This can be explained by the different amount of stretching of tracer as shown in the Figs. 3cd at day 180.

The stirring efficiency $\zeta$ in (15) (Fig. 14) is shown as a function of equivalent deviation, $\sigma_{e}$. In theory, $\zeta$ should be at least 1 since effective diffusivity cannot be smaller than the background diffusivity. In our models, this is only true after a certain number of days since initially there is no substantial straining/stretching of the tracer contours and so the tracer patch is small with large gradients at the grid scale. For the $1 / 12^{\circ}$ run $\zeta$ is consistently greater than 1 after 70 days and for the $1 / 4^{\circ}$ run after 285 days. Therefore, we can set the time when $\zeta \geq 1$ to be the time when straining by shear flows begins to operate. This time scale also corresponds well with the time scales when the growth rate of equivalent variance switches from linear to faster than linear.

In the following, we attempt to explain why the stirring efficiency is 10 times larger in the $1 / 12^{\circ}$ run than in the $1 / 4^{\circ}$ run (Fig. 14). If we scale $\bar{\kappa}_{e} \sim \kappa_{a} \sim \sqrt{\lambda k} \sigma_{e}$ (from section 5.1), then $\zeta \equiv \bar{\kappa}_{e} / k \sim$ $\sqrt{\lambda / k} \sigma_{e}$. This implies that at a given equivalent deviation, $\sigma_{e}$, the stirring efficiency is scaled as 
$\sqrt{\lambda / k}$, representing the competition between stirring and mixing. In our experiments, the ratio of $\sqrt{\lambda / k}$ between the $1 / 12^{\circ}$ run and the $1 / 4^{\circ}$ is about 6.3 .

As stated in section 2.4, the true advantage of $\zeta$ is that it can be calculated directly from the tracer fields without needing to estimate strain rate or background diffusivity. This may be useful as a way to separate different flow regimes in the ocean by applying to tracers in the different locations. To get some idea of the order of magnitude of $\zeta$ in the ocean, we can in theory estimate $\zeta$ from $k_{h} / k$. Assume $k_{h} \sim 10^{3} \mathrm{~m}^{2} \mathrm{~s}^{-1}$, then the stirring efficiency in the real ocean could vary from $\sim 10^{4}$ to 500, depending what background diffusivity is considered (according to Lewell et al. 1998, $k=0.07$ $\mathrm{m}^{2} \mathrm{~s}^{-1}$ for the scale $0.1-1 \mathrm{~km}$ and $k=2 \mathrm{~m}^{2} \mathrm{~s}^{-1}$ for the scale $1-10 \mathrm{~km}$ ).

\subsection{Comparing to the apparent diffusivity}

The apparent and mean effective diffusivities plotted together in Fig. 9 shows that $\kappa_{a}$ is twice as large as $\bar{\kappa}_{e}$. As explained in section 2.6, these two diffusivities are two different ways of averaging effective diffusivity $\kappa_{e}$. Here, we illustrate this difference using an example from EXP1/12 at day 730. The diffusivity $\bar{\kappa}_{e}$ is an average weighted by the squared tracer gradient in equivalent radius coordinate. It puts more weight toward the centre of the tracer patch where tracer contours are bunched together and so tracer gradients are larger (dashed line in the upper panel of Fig. 15a). Near the center of the patch the effective diffusivity is relatively smaller (solid line in Fig. 15 a) and so the result of tracer gradients weighting is a nearly uniform diffusivity across the tracer contours (dotted line in Fig. 15a). In this example, while $\kappa_{e}$ increases from 0 near the centre of the tracer patch to $2500 \mathrm{~m}^{2} \mathrm{~s}^{-1}$ toward outer edge, the mean diffusivity $\bar{\kappa}_{e}$ is only about $450 \mathrm{~m}^{2} \mathrm{~s}^{-1}$, which is about the value of diffusivity at the tracer contour containing $10 \%$ of the tracer load.

This may be compared to a simpler average where the weighting is just tracer concentration rather than tracer gradients, $\kappa_{s}=\int \kappa_{e} \hat{C} d a / \int \hat{C} d a$. This average puts more weight away from the centre of the tracer patch (dashed line in Fig. 15b). As a result, the weighted diffusivity maintains a similar shape as the un-weighted one and the averaged value is about $1400 \mathrm{~m}^{2} \mathrm{~s}^{-1}$, which is about the value of diffusivity at the contour containing $50 \%$ of tracer load.

For the apparent diffusivity, the quantity to be averaged is $\frac{\partial\left(\kappa_{e} \mathcal{A}\right)}{\partial \mathcal{A}}$. Although it varies the same way as $\kappa_{e}$, it has a much larger value towards the outer edge of tracer patch (compare solid lines in Fig. 
15bc). This is because $\kappa_{e}$ is not constant - it increases toward the outer edge. The weighting for $\kappa_{a}$ also put more weight near the outer edge of the tracer and so the combination gives a value $\kappa_{a}$ about $1900 \mathrm{~m}^{2} \mathrm{~s}^{-1}$. This is about the value of diffusivity at the contour containing $75 \%$ of tracer load.

Using the tracer contour, $C_{.65}$, enclosing $65 \%$ tracer load as a guide, the mean effective diffusivity $\bar{\kappa}_{e}$ puts $93 \%$ of weight inside the contour $C_{.65}$, whereas the apparent diffusivity $\kappa_{a}$ by definition has exactly $65 \%$ of weight inside the $65 \%$ contour. The problem with $\kappa_{a}$ is that the quantity to be averaged is twice of the original $\kappa_{e}$ which leads to a much larger average value. Intuitively, the simple average $\kappa_{s}$ seems to give an fairly representative average, although it is not clear how to link $\kappa_{s}$ with some equation to give it a physical meaning.

\section{The possible effect of tracer loss on estimating diffusivity}

The tracer in the model is not conserved on the target density layer. The transfer of tracer across density layers can be due to both vertical diffusivity (explicit and implicit) and the horizontal advection scheme. In order to separate the effect of each process, the details of tracer budget at each grid cell would need to be calculated which we are not able to do for this study. However, there are clues that may help us to estimate the likely impact of diapycnal processes on our results.

To give an estimate, we consider an simple approximation where the loss of tracer substance is proportional to the tracer concentration (e.g. assume the tracer concentration on either side of the target density layer is negligible and so higher tracer concentration corresponds to larger vertical tracer gradient and larger diffusive flux divergence):

$$
\dot{C} \sim-\mu C
$$

where $\dot{C}$ is diapycnal flux of tracer across a density layer and $1 / \mu$ is an spatially averaged time scale. In reality, $\mu$ could vary from point to point. From (19), we estimate the additional tracer loss term in the variance equation (11) as

$$
\mathcal{S}=\int \dot{C} C d a \sim-\mu \int C^{2} d a=-C^{*} \int \dot{C} d a,
$$

where $C^{*}=\frac{\int C^{2} d a}{\int C d a}$ is the mean tracer concentration in the density layer. Using the r.h.s. of (20), the tracer loss term $\mathcal{S}$ can be calculated using the two integral quantities, the mean tracer concentration, $C^{*}$, and the total loss of tracer substance in the density layer, $\int \dot{C} d a$. 
Thus, an adjusted effective diffusivity including the effect of the tracer loss term can be defined as $\bar{\kappa}_{e}^{\text {adjust }}=\bar{\kappa}_{e}+\bar{\kappa}_{e}^{S}$ where $\bar{\kappa}_{e}^{S}=\mathcal{S} / \int\left|\frac{\partial \hat{C}\left(\gamma_{e}, t\right)}{\partial \gamma_{e}}\right|^{2} d a$. Similarly, the adjusted mean background diffusivity is $\bar{\kappa}^{\text {adjust }}=\bar{\kappa}+\bar{\kappa}^{S}$ where $\bar{\kappa}^{S}=\mathcal{S} / \int|\nabla C|^{2} d a$.

The adjusted effective diffusivity $\bar{\kappa}_{e}^{\text {adjust }}$ is shown by the dotted lines in Fig. 11 . For the $1 / 12^{\circ}$ run, the tracer loss term is about $16 \%$ of the rate of change of the tracer variance. The adjustment for the effective diffusivity $\bar{\kappa}_{e}^{S}$ is $-82 \pm 88 \mathrm{~m}^{2} \mathrm{~s}^{-1}$. For mean background diffusivity, the adjustment is about $\bar{\kappa}^{S}=-3.6 \pm 2.9 \mathrm{~m}^{2} \mathrm{~s}^{-1}$ (not shown). For the $1 / 4^{\circ}$ run, the tracer loss term is about $14 \%$ of the rate of change of the variance. This corresponds to the adjustment of $\bar{\kappa}_{e}^{S}=-16.9 \pm 17 \mathrm{~m}^{2} \mathrm{~s}^{-1}$ to the effective diffusivity and $\bar{\kappa}^{S}=-6.9 \pm 5 \mathrm{~m}^{2} \mathrm{~s}^{-1}$ to the background diffusivity.

How does the diapycnal diffusion affect the equivalent variance ? Take the solution for a point release tracer in a three dimensional uniform-shear flow with $u=u_{0}+\lambda_{y} y+\lambda_{z} z, v=w=0$ (where $\lambda_{y, z}$ are the constant shears in the $y$ and $z$ direction) (Okubo 1968). For a time long enough that the stirring dominates the background diffusion, the mean variance on a horizontal slice can be approximated as $\sigma_{x} \sigma_{y} \sim k_{y} \lambda_{y} t^{2} \sqrt{1+\frac{1}{4}\left(\frac{\lambda_{z}}{\lambda_{y}}\right)^{2} \frac{k_{z}}{k_{y}}}$ (where $k_{y, z}$ are the constant diffusivities in the $y$ and $z$ direction). This implies that the variance can be affected by the combination of vertical shear of horizontal flow and vertical diffusivity, i.e. the tracer is diffused vertically to different levels and advected horizontally before being diffused vertically back to the original level. Let $\lambda_{z} / \lambda_{y} \sim L_{y} / L_{z} \sim 3 \times 10^{2}$ (where $L_{y} \sim 100 \mathrm{~km}$ and $L_{z} \sim 300 \mathrm{~m}$ are the horizontal and vertical length scale of eddies) and $k_{z} / k_{y} \sim 10^{-6}$ (where $k_{z} \sim 0.1 \mathrm{~cm}^{2} \mathrm{~s}^{-1}$ and $k_{y} \sim 10 \mathrm{~m}^{2} \mathrm{~s}^{-1}$ are the estimates of model vertical and horizontal numerical diffusivities). Using these estimates, the additional term would change the mean variance by $1 \%$. This implies that in our model the influence of vertical shear/diffusivity is small. It would not be unreasonable to expect the equivalent variance to have a similar behaviour. For the real ocean, if take $k_{y}=2 \mathrm{~m}^{2} \mathrm{~s}^{-1}$ for the scale 1 to $10 \mathrm{~km}$, then the vertical shear term can alter variance by $5 \%$. If take $k_{y}=0.07 \mathrm{~m}^{2} \mathrm{~s}^{-1}$ for the scale 0.1 to $1 \mathrm{~km}$ then the ratio $k_{z} / k_{y}$ becomes much larger. However, at this scale eddies becomes smaller so the ratio $\lambda_{z} / \lambda_{y}$ becomes smaller. Thus, it is not clear that at the scale of 0.1 to $1 \mathrm{~km}$ what would be the impact of the vertical shear/diffusiviity on the variance over a horizontal slice. Futher investigation is needed in order to properly quantify the effect of vertical processes on the variance. This is however beyond the scope of our study. 


\section{Summary}

Lagrangian observations such as tracers and floats are frequently used to estimate eddy diffusivity. Because of the sparse spatial-temporal coverage of the data, these estimates are inevitably uncertain. In addition, the relationship between different methods of inferring diffusivity is often not clear which makes it difficult to interpret the meaning of eddy diffusivity. This study examined two diagnostic methods that are applied to point release tracers.

The tool used in this study is based on the concept of equivalent radius, $\gamma_{e}$. It allows us to bypass $x$ and $y$ coordinates and to concentrate on the intrinsic nature of tracer dispersion. The variance of equivalent radius, $\sigma_{e}^{2}$, for example, is related to the average of area enclosed by tracer contours. The apparent diffusivity, $\kappa_{a}$, is thus defined as the growth rate of equivalent variance. The initial tracer dispersion is dominated by small-scale background diffusion with $\sigma_{e}^{2}$ increasing linearly. From this, we diagnose a mean numerical diffusivity associated model's advection scheme to be about 10-15 $\mathrm{m}^{2} \mathrm{~s}^{-1}$. After the initial diffusion stage, the tracer dispersion is dominated by the stirring due to shear flows. In our $1 / 12^{\circ}$ run the growth rate of equivalent variance during this stage is between the pure shearing flow regime (power of 2 growth) and the pure stretching flow regime (exponential growth). In the ocean, earlier studies suggested that the variance increases with time between $2^{\text {nd }}$ and $3^{r d}$ power law (Okubo 1971) and more recent result from NATRE also found a close to $2^{\text {nd }}$ power law (Ledwell et al. 1998).

Another way of using equivalent radius is to use it as a coordinate to transform the advection-diffusion tracer equation (Nakmura 1996). The result is a simple diffusion equation with an effective diffusivity $\kappa_{e}=\kappa L_{e q}^{2} / L_{0}^{2}$. As a tracer contour is distorted by shear flows, the available interface for small-scale diffusion increases and so gives a higher value of effective diffusivity. In this context, effective diffusivity is a function of tracer contours and it reflects the geometrical complexity of tracer distribution regardless of the history of the tracer dispersion.

We take a step further to propose a new way of evaluating $\kappa_{e}$ by relating the diffusive flux of tracer across tracer contours to the gradients of tracer w.r.t the area enclosed by tracer contours. In this way, the effective diffusivity has a physical meaning (the flux-gradient relationship) in addition to the geometric one. The new way of calculating $\kappa_{e}$ also has the advantage that it does not require prior knowledge of background diffusivity, which is difficult to obtain. On the other hand, it does require 
tracer fields between short time intervals which may be difficult to acquire for tracers released in the ocean.

Previous studies (e.g. Allen and Nakamura 2001, Marshall et al. 2006) applied effective diffusivity diagnosis to quasi-steady tracers. For such tracers, the tracer contours can be associated with geographical locations and so the spatial pattern of effective diffusivity can be linked to the spatial characteristics of the flow such as the region of strong mixing. A point release tracer is always evolving in time and so it is not meaningful to follow a given tracer contour. Thus, we introduce a new quantity called the mean effective diffusivity $\bar{\kappa}_{e}$ which assigns an effective diffusivity at any instant time for the whole tracer patch rather than for individual tracer contours. It also represents an average of $\kappa_{e}$ weighted by the tracer gradients, c.f. (13).

The mean effective diffusivity $\bar{\kappa}_{e}$ may be compared to the apparent diffusivity $\kappa_{a}$. First we interpret the apparent diffusivity as the tracer-weighted average of a quantity that takes into account variations of $\kappa_{e}$ across tracer contours, c.f. (18). So, the mean effective diffusivity $\bar{\kappa}_{e}$ (based on the tracer variance, $\int C^{2}$ ) and the apparent diffusivity $\kappa_{a}$ (based on the equivalent variance, $\sigma_{e}^{2}$ ) are in fact two different ways of averaging $\kappa_{e}$. It is not clear which averaging is more meaningful except that $\bar{\kappa}_{e}$ represents an average that puts more weight over the area inside a tracer contour containing $65 \%$ of total tracer load. The two diffusivities have a similar value only when tracer dispersion reaches the final diffusion stage.

A more traditional approach is to infer an overall eddy diffusivity from the evolution of variance at a later time. The idea is that during the final stage of tracer dispersion the variance converges to a linear growth rate (Garrett 1983). In our models, after three years the traditional distance (to the center of mass) variance $\sigma_{r}^{2}$ seems to approach a linear growth stage which would give an overall eddy diffusivity $k_{h}=1000 \mathrm{~m}^{2} \mathrm{~s}^{-1}$. However, the equivalent variance $\sigma_{e}^{2}$ has not reached the final diffusion stage. We estimate it would take about 5 or 6 years for $\sigma_{e}^{2}$ to converge to linear growth. The point is that distance-based variance, e.g. $\sigma_{r}^{2}$, always reaches the final linear growth stage sooner than area-based variance, e.g. $\sigma_{e}^{2}$. So, when inferring diffusivity one needs to be cautious and note that the time scale associated the final stage of tracer evolution is different for different methods.

In summary, the apparent diffusivity, $\kappa_{a}$, and the effective diffusivity, $\bar{\kappa}_{e}$, represent different averagings over the tracer patch. Over a longer time when tracer contours are merged and the tracer patch 
is nearly Gaussian, all three diffusivities $k_{h}, \kappa_{a}$ and $\bar{\kappa}_{e}$ are expected to converge to a similar value. In our experiment, this value could be about $1000 \mathrm{~m}^{2} \mathrm{~s}^{-1}$.

The tracer dispersion is ultimately an interplay between the stirring due to shear and mixing due to background diffusion. In our opinion, the fundamental quantity is the equivalent radius (which gives rise to the equivalent variance $\sigma_{e}^{2}$ and the equivalent length $L_{e}$ ). The effective diffusivity is a secondary quantity as it is not uniquely defined (e.g. $\kappa_{e}, \kappa_{a}$ and $k_{h}$ ) and during the stirring-dominating stage the diffusivity will be time dependent. If the radius of a tracer patch is scaled as $\sigma_{e}$, then the rate of increase of the radius of tracer is scaled $\frac{\partial \sigma_{e}}{\partial t} \sim \sqrt{\lambda k}$, indicating that stirring and mixing work together to increase the size of tracer patch.

We have introduced a new diagnostic quantity called the stirring efficiency, $\zeta$. It simply measures the degree of deformation (in terms of gradients) of the tracer patch relative to it otherwise would be without shear flows. Thus, it is an indicator of how efficient the stirring is against mixing. Like Péclet number, stirring efficiency characterises the flows in terms of advection versus diffusion. However, the advantage of $\zeta$ is that it can be calculated from tracer fields without needing to estimate the length scale of flow or the background diffusivity. The stirring efficiency may be scaled as $\zeta \sim \sqrt{\lambda / k} \sigma_{e}$, which is consistent with the intuition that the efficiency of stirring is a result of the competitions between stirring and mixing.

The stirring efficiency may be useful as a way of assessing models' simulations of tracer evolution. For example, the comparison between the stirring efficiency for the tracers in the $1 / 12^{\circ}$ and $1 / 4^{\circ}$ runs suggests that the $1 / 12^{\circ}$ model is about 10 times more efficient than the $1 / 4^{\circ}$ model in terms of stirring tracers. If the stirring efficiency in the real ocean is estimated to be about $10^{2}-10^{4}$, depending on the length scale considered for mixing, then much lower values of stirring efficiency in the models indicates the deficiency of the models. Such deficiency may be due to model's large numerical diffusion (e.g. the $1 / 12^{\circ}$ run), or due to the combination of weak strain rate and large explicit diffusion (e.g. the $1 / 4^{\circ}$ run).

This leads to the question of what impact the $50 \mathrm{~m}^{2} \mathrm{~s}^{-1}$ explicit isopycnic diffusivity in the $1 / 4^{\circ}$ model has on the tracer simulation. From the perspective of equivalent variance $\sigma_{e}^{2} \sim \lambda k t^{2}$, the explicit isopycnic diffusivity compensates for the smaller strain rate. For example, the diffusion velocity $\sqrt{\lambda k}$ in the $1 / 4^{\circ}$ model is half of that in the $1 / 12^{\circ}$ model rather than ten times smaller as it would 
be implied by the strain rate alone. Thus, one can argue larger isopycnic diffusivity is necessary in order to rectify smaller strain rate. On the other hand, the stirring efficiency is greatly reduced with the presence of explicit diffusion (as explained in the previous paragraph). Thus, one might argue that smaller explicit isopycnic diffusivity for the $1 / 4^{\circ}$ model is preferrable. On top all these, it is not clear to what degree the explicit isopycnic diffusivity affects the strain rate in the $1 / 4^{\circ}$ model. Since the time scale for the onset of stirring-domination is determined by the strain rate, it would always take longer for the $1 / 4^{\circ}$ tracer to catch up to the same effective diffusivity in the $1 / 12^{\circ}$ model. Even when tracers in the two model resolutions give a similar value of effective diffusivity, they will be achieved through different mechanisms. For example, we see in the Figs. 4 and 5 that tracer in the $1 / 12^{\circ}$ model (day 365 ) has fine filaments with pinch-off whereas tracer in the $1 / 4^{\circ}$ model (day 735) remains as a coherent structure. It remains a difficult issue to decide what values of isopycnic diffusivity should be used in the lower resolution model. The answers depend on the criteria for assessing model performance.

\section{Acknowledgements}

We thank Alberto Naveira Garabato for the motivations and discussions of this work. We are especially grateful to the referees whose constructive comments on the earlier drafts helped to improve the manuscript.

\section{Appendix}

\section{A The transformed tracer equation}

Here, we give a layer formulation and all our diagnostics are done in the layer format accordingly. The evolution equation for a passive tracer in an isopycnal layer between isopycnals $\sigma$ and $\sigma+\delta \sigma$ is

$$
\frac{\partial(\tau h)}{\partial t}+\nabla \cdot(\mathbf{u} h \tau)+\frac{\partial}{\partial \sigma}(\dot{\sigma} h \tau)=\dot{\tau} h,
$$

where $\tau$ is the tracer concentration, $h$ is the isopycnal layer thickness per unit of density, $\nabla$ is the isopycnal gradient, and $\mathbf{u}$ is the isopycnal velocity. $\dot{\tau} \equiv d \tau / d t$ and $\dot{\sigma} \equiv d \sigma / d t$ are the material derivatives of $\tau$ and $\sigma$, respectively, representing diffusion and source/sink. 
Following Nakamura (1995), a control volume bounded by a tracer contour $\tau$ and lying between isopycnals $\sigma$ and $\sigma+\delta \sigma$ can only be changed through the nonadvective nature of tracer flux across the tracer contour and through the nonadvective nature of density flux across isopycnals. This is expressed as

$$
\frac{\partial}{\partial t} \int_{\mathcal{A}_{\tau}} h d a+\frac{\partial}{\partial \tau} \int_{\mathcal{A}_{\tau}} \dot{\tau} h d a+\frac{\partial}{\partial \sigma} \int_{\mathcal{A}_{\tau}} \dot{\sigma} h d a=0
$$

where $d a$ is the area element and $\mathcal{A}_{\tau}$ is the area inside the tracer contour $\tau$.

For simplicity, we assume that cabelling and diffusive density flux may be ignored $(\dot{\sigma} \sim 0)$. Thus, the volume can only be changed through nonadvective process acting on the tracer,

$$
\frac{\partial}{\partial t} \int_{\mathcal{A}_{\tau}} h d a=-\frac{\partial}{\partial \tau} \int_{\mathcal{A}_{\tau}} \dot{\tau} h d a
$$

Since there is no forcing of the tracer, $\dot{\tau}$ can be only due to diffusive flux of tracer. This diffusive flux consists of the horizontal divergence of horizontal diffusive flux and the vertical divergence of diapycnal component of diffusive flux,

$$
\dot{\tau} h=-\nabla \cdot\left(\mathbf{D}_{\mathbf{2}} h\right)-\frac{\partial}{\partial \sigma}(\mathbf{D} \cdot \mathbf{n}),
$$

where $\mathbf{D}=-\kappa \nabla_{3} \tau$ is the three-dimensional diffusive flux in $(x, y, z)$ coordinate, $\mathbf{D}_{\mathbf{2}}$ is the horizontal component of $\mathbf{D}, \kappa$ is the background diffusivity which can be either molecular diffusivity (in the real ocean) or numerical diffusivity (in models) and $\mathbf{n}$ is the unit vector normal to the isopycnal.

For simplicity, we further assume the diapycnal component, $\mathbf{D} \cdot \mathbf{n}$, is small compared to the horizontal component. Thus, from (23) and (24), we have

$$
\frac{\partial}{\partial t} \int_{\mathcal{A}_{\tau}} h d a=-\frac{\partial}{\partial \tau} \int_{\mathcal{A}_{\tau}} \nabla \cdot(\kappa h \nabla \tau) d a=\frac{\partial}{\partial \tau} \oint_{\tau} \kappa|\nabla \tau| h d l=-\frac{\partial}{\partial \tau} \frac{\partial}{\partial \tau} \int_{\mathcal{A}_{\tau}} \kappa|\nabla \tau|^{2} h d a,
$$

where $d l$ is the line element and so $d a=d l d \tau /|\nabla \tau|$. The second equality uses Gauss' theorem. The third equality uses the identity

$$
\oint_{\tau}(\cdot) h \frac{d l}{|\nabla \tau|}=-\frac{\partial}{\partial \tau} \int_{\mathcal{A}_{\tau}}(\cdot) h d a
$$

Note that the sign convention is such that $\tau$ increases towards the centre of the tracer patch, as it would be for a point-released tracer.

To really appreciate the essence of tracer-based coordinates, we need to use the volume enclosed by the contours of tracer as a coordinate. Define $\mathcal{V}_{\tau}=\int_{\mathcal{A}_{\tau}} h d a$ and $\tau(\mathcal{V}, t)$ such that $\tau\left(\mathcal{V}_{\tau^{*}}, t\right)=\tau^{*}(t)$. 
Following Nakamura (1996) and use the equality $\frac{\partial \tau(\mathcal{V}, t)}{\partial t}=-\frac{\partial \tau(\mathcal{V}, t)}{\partial \mathcal{V}} \frac{\partial \mathcal{V}}{\partial t},(25)$ can be transformed to

$$
\frac{\partial \tau(\mathcal{V}, t)}{\partial t}=\frac{\partial}{\partial \mathcal{V}}\left(\kappa L_{e q}^{2} \frac{\partial \tau(\mathcal{V}, t)}{\partial \mathcal{V}}\right)
$$

where

$$
L_{e q}^{2}=\oint_{\tau} \frac{1}{|\nabla \tau|} h d l \oint_{\tau}|\nabla \tau| h d l
$$

In (27), $\kappa L_{e q}^{2}$ does not yet have units of diffusivity. To make it more transparent, we use 'equivalent radius' instead of volume as a coordinate. For simplicity, we assume the region of interest is on a flat plane rather than on a sphere. For a given volume $\mathcal{V}$, define the 'equivalent radius' $\gamma_{e q}$ to be such that $\mathcal{V}=\pi \gamma_{e q}^{2} \bar{h}$ where $\bar{h}$ is the mean isopycnal layer thickness for $\mathcal{V}$.

\section{B The strain rate and stretch rate}

The strain rate in the model was calculated using two different expressions: the rms of strain rate $\lambda=\partial u / \partial y+\partial v / \partial x$, representing the shearing deformation, and the rms of stretching rate $\eta=$ $\partial u / \partial x-\partial v / \partial y$, representing the extension (or contraction) deformation. The rms is the average over the region occupied by tracer.

The three annual mean strain rates in units of $10^{-6} \mathrm{~s}^{-1}$ are $1.18,0.77,0.88$ for the $1 / 12^{\circ}$ run and 0.08 , $0.12,0.17$ for $1 / 4^{\circ}$ run. The annual mean stretching rates in the same units are $0.95,0.75,0.85$ for the $1 / 12^{\circ}$ run and $0.08,0.12,0.16$ for $1 / 4^{\circ}$ run.

\section{References}

Allen, D. R., and N. Nakamura, 2001: A seasonal climatology of effective diffusivity in the stratophere. J. Geophy. Res., 106, 7917-7935.

Coward, A. C., and B. A. de Cuevas, 2005: The OCCAM 66 level model: physics, initial conditions and external forcing. SOC internal report No. $99,58 \mathrm{pp}$.

Garrett, C., 1983: On the initial streakiness of a dispersing tracer in two- and three-dimensional turbulence, Dyn. Atmos. Oceans, , 7, 265-277.

Garrett, C., 2006: Turbulent dispersion in the ocean. Progr. Ocean., 70, 113-125.

Joseph, J., and H. Sender, 1958: Uber die horizontale diffusion im Meere. Dt. hydrogr. Z., 11, 49-77. 
Ledwell, J. R., A. J. Watson, and C. S. Law, 1998: Mixing of a tracer in the pycnocline. J. Geophy. Res., 103, 21499-21529.

Ledwell, J. R., E. T. Montgomery, K. L. Polzin, L. C. St. Laurent, R. W. Schmitt, and J. M. Toole, 2000: Evidence for enhanced mixing over rough topography in the abyssal ocean. Nature, 403, 179-182.

Lee, M.-M., A. C. Coward, and A. J. G. Nurser, 2007: Spurious diapycnal mixing of the deep waters in an eddy-permitting global ocean model J. Phys. Oceanogr., 32, 1522-1535.

Lee, M.-M., A. J. G. Nurser, A. C. Coward, and B. A. de Cuevas, 2007: Eddy advective and diffusive transports of heat and salt in the Southern Ocean. J. Phys. Oceanogr., 37, 1376-1393.

Marshall, J., E. Shuckburgh, H. Jones, and C. Hill, 2006: Estimates and implications of surface eddy diffusivity in the Southern Ocean derived from tracer transport. J. Phys. Oceanogr., 36, 1806-1821.

Nakamura, N., 1995: Modified Lagrangian-mean diagnostics of the stratospheric polar vortices. 1. Formulation and analysis of GFDL SKYHI GCM. J. Atmos. Sci., 52, 2096-2108.

Nakamura, N., 1996: Two-dimensional mixing, edge formation annd permeability diagnosed in an area coordinate. J. Atmos. Sci., 53, 1524-1537.

Novokov, E. A., 1958: Concerning a turbulent diffusion in a stream with a transverse gradient of velocity. J. Appl. Math. and Mech., 22, 576-579.

Okubo, A., 1966: A note on horizontal diffusion from an instantaneous source in a nonuniform flow. J. Oceanogr. Soc. Japan, 22, 35-40.

Okubo, A., 1968: Some remarks on the importance of the "shear effect" on the horizontal diffusion. J. Oceanogr. Soc. Japan, 24, 60-69.

Okubo, A., 1971: Oceanic diffusion diagrams Deep-Sea Res., 18, 789-802.

Sanderson, B. G., and A. Okubo, 1986: An analytical calculation of two-dimensional dispersion. $J$. Oceanogr. Soc. Japan, 42, 139-153.

Schmitt, R. W., J. R. Ledwell, E. T. Montgomery, K. L. Polzin, and J. M. Toole, 2005: Enhanced 
diapycnal mixing by salt fingers in the thermocline of the tropical Atlantic. Science, 308, 685-688.

Shuckburgh, E., and P. Haynes, 2003: Diagnosing transport and mixing using a tracer-based coordinate system. Phys. Fluids, 15, 3342-3357.

Sundermeyer, M. A., and J. F. Price, 1998: Lateral mixing and the North Atlantic Tracer Release Experiment: Observations and numerical simulations of Lagrangian particles and a passive tracer. $J$. Geophy. Res., 103, 21481-21497.

Townsend, A. A., 1951: The diffusion of heat spots in isotropic turbulence. Proc. Roy. Soc. London, A209, 418-430.

Watson, A. J., M. J. Messias, E. Fogelqvist, K. A. Van Scoy, T. Johannessen, K. I. C. Oliver, D. P. Stevens, F. Rey, T. Tanhua, K. A. Olsson, F. Carse, K. Simonsen, J. R. Ledwell, E. Jansen, D. J. Cooper, J. A. Kruepke, and E. Gullyardl, 1999: Mixing and convection in the Greenland Sea from a tracer-release experiment. Nature, 401, 902-904.

Webb, D.J., B.A. de Cuevas and C.S. Richmond, 1998b: Improved advection schemes for ocean models. J. Atmos. Ocean. Tech., 15, 1171-1187.

Young, W. R., P. B. Rhines, and C. J. R. Garrett, 1982: Shear flow dispersion, internal waves and horizontal mixing in the ocean. J. Phys. Oceanogr., 12, 515-527. 


\section{Figure captions}

Fig.1

The shading gives the mean eddy kinetic energy $\left(\mathrm{cm}^{2} \mathrm{~s}^{-1}\right)$ at depth $314 \mathrm{~m}$ (model level 25) for year 1995 from the $1 / 12^{\circ}$ run. The highest eddy kinetic energy (indicated by darker shading) is in the region of the Gulf Stream. The contours (in units of $10^{4} \mathrm{~Pa}$ ) gives the mean pressure field at the same depth for the same year. The triangle indicates the site of tracer release in the model and the rectangular box corresponds to the plots shown in Figs. 2-5.

Fig.2

Five-day mean fields from the $1 / 12^{\circ}$ run. The left panels (a) and (b) are the pressure fields at 314 m. The color scale is on the left with the units of $10^{4} \mathrm{~Pa}$ and contour interval is $80 \mathrm{~Pa}$. (a) is at the time of tracer release for EXP1/12 $2_{\text {test }}$. (b) is at the time of tracer release for EXP1/12. The right panels (c) and (d) are the tracer concentration on the target density layer after the 180 days (each right panel corresponds to the release on the left panel). The tracer concentration is normalised to have the maximum value 1 . The color scale is on the right with the magenta color showing the negative tracer value. The release site is marked by the triangle. For comparison, the domain of plots is also drawn in Fig.1.

Fig. 3

As in Fig. 2, but for the $1 / 4^{\circ}$ run. The tracer fields on the right panels are 360 days after releases.

Fig. 4

For EXP1/12. The upper panels are the normalised tracer concentration on the target density layer (color scale on the left). The lower panels are effective eddy diffusivity $\kappa_{e}\left(\mathrm{~m}^{2} \mathrm{~s}^{-1}\right)$ (color scale on the right). The left, middle and right panels correspond to day 180, 365 and 730 days, respectively.

Fig. 5

As in Fig. 4 but for EXP1/4.

Fig. 6 
The three years time series of areas from EXP1/12: from the equivalent variance, $\pi \sigma_{e}^{2}$ (thick solid line), from the conventional distance variance, $\pi \sigma_{r}^{2}$ (dashed line), the tracer area containing $65 \%$ tracer load (dotted line), the tracer area containing 95\% tracer load (dot-dashed line), the tracer area, $\Gamma$ (thin solid line).

Fig. 7

Left panels: the time series of the equivalent variance, $\sigma_{e}^{2}$ (thick solid lines), in log-log scale for the run of (a) $1 / 12^{\circ}$ and (b) $1 / 4^{\circ}$. The three triangles mark 180 days, 1 year and 2 years. The thin solid lines show $\sigma_{e}^{2}(0)+4 k t$ (where $k=15$ and $60 \mathrm{~m}^{2} \mathrm{~s}^{-1}$ in $1 / 12^{\circ}$ and $1 / 4^{\circ}$ run, respectively) (thin solid lines). The $\sigma_{e}^{2}(0)$ is the variance at the time of release. Note the thin and solid lines almost overlap. Also plotted are curves of the $2^{\text {nd }}$ power law, $\lambda k t^{2}$ (dashed lines), and the exponential law, $\sigma_{e}^{2}(T) e^{0.2 \eta(t-T)}$ (dotted lines), relevent to the stirring-dominated stage. $\sigma_{e}^{2}(T)$ is the value of $\sigma_{e}^{2}$ at the time $T$ (70 days and 360 days for $1 / 12^{\circ}$ and $1 / 4^{\circ}$ runs, respectively). See text for the values of straining rate $\lambda$ and stretching rate $\eta$. Right panels: the conventional distance variance, $\sigma_{r}^{2}$ (thick solid line). The superimposed line in EXP1/12 is $12 k_{h} t$, where $k_{h}=1000 \mathrm{~m}^{2} \mathrm{~s}^{-1}$ (thin solid line).

Fig. 8

The time series of the equivalent deviation $\sigma_{e}$ from EXP1/12 (solid line) and EXP1/4 (dashed line).

Fig. 9

The time series of apparent diffusivity $\kappa_{a}$ (solid line) (smoothed with 30-day filter) and mean effective diffusivity $\bar{\kappa}_{e}$ (dashed line) from EXP1/12.

Fig. 10

The time series of apparent diffusivity $\kappa_{a}\left(\mathrm{~m}^{2} \mathrm{~s}^{-1}\right)$ plotted as a function of equivalent deviation $\sigma_{e}$ for EXP1/12 (solid line) and EXP1/4 (dashed line).

Fig. 11

The diffusivity $\left(\mathrm{m}^{2} \mathrm{~s}^{-1}\right)$ over three years. The left panel is for the $1 / 12^{\circ}$ run and the right panel is for the $1 / 4^{\circ}$ run. The background diffusivity $k$ is given by the dashed lines and the mean effective diffusivity $\bar{\kappa}_{e}$ is given by the solid lines. The thick lines are from the main runs and thin lines are 
from the test runs. The dotted lines are $\bar{\kappa}_{e}^{\text {adjust }}=\bar{\kappa}_{e}+\bar{\kappa}_{e}^{S}$.

Fig. 12

The diffusivity $\left(\mathrm{m}^{2} \mathrm{~s}^{-1}\right)$ as a function of equivalent radius $\gamma_{e}(\mathrm{~km})$. The dashed lines are the background diffusivities. The dotted and the solid lines are the effective diffusivities calculated from (8) and (10), respectively. The left panel is from EXP1/12 at day 365 and the right panel is from EXP1/4 at day 730 .

Fig. 13

Length for the contours of tracer from EXP1/12 at day 365 as a function of equivalent radius $\gamma_{e}$ plotted in log-log scale: the equivalent length $L_{e}$ (thick solid line), the actual length $L$ (dashed line), the minimal length $L_{0}=2 \pi \gamma_{e}$ (thick dotted line). The superimposed lines are $\gamma_{e}^{2}$ (thin solid line) and $L_{e} / L_{0}$ (thin dotted line).

Fig. 14

The eddy efficiency $\zeta$ plotted against equivalent deviation $\sigma_{e}$. The solid line is from EXP1/12 and the dashed line is from EXP1/4.

Fig. 15

Quantities contribute to mean diffusivities at day 730 of EXP1/12. (a) mean effective diffusivity $\bar{\kappa}_{e}$, (b) for comparison $\kappa_{s}=\int \kappa_{e} C d a / \int C d a$ and (c) apparent diffusivity $\kappa_{a}$. The $x$-axis is the normalised tracer load encompassed by tracer contours with 1 corresponding to total tracer load enclosed by the lowest tracer concentration. In (a), $\kappa_{e}$ (solid line), weighting $\left|\frac{\partial \hat{C}}{\partial \gamma_{e}}\right|^{2} d a / \int\left|\frac{\partial \hat{C}}{\partial \gamma_{e}}\right|^{2} d a$ (dashed line) and the multiplication of the two (dotted line). In (b), $\kappa_{e}$ (solid line), weighting $C d a / \int C d a$ (dashed line) and the multiplication of the two (dotted line). In (c), $\frac{\partial}{\partial \mathcal{A}}\left(\kappa_{e} \mathcal{A}\right)$ (solid line), weighting $C d a / \int C d a$ (dashed line) and the multiplication of the two (dotted line). 


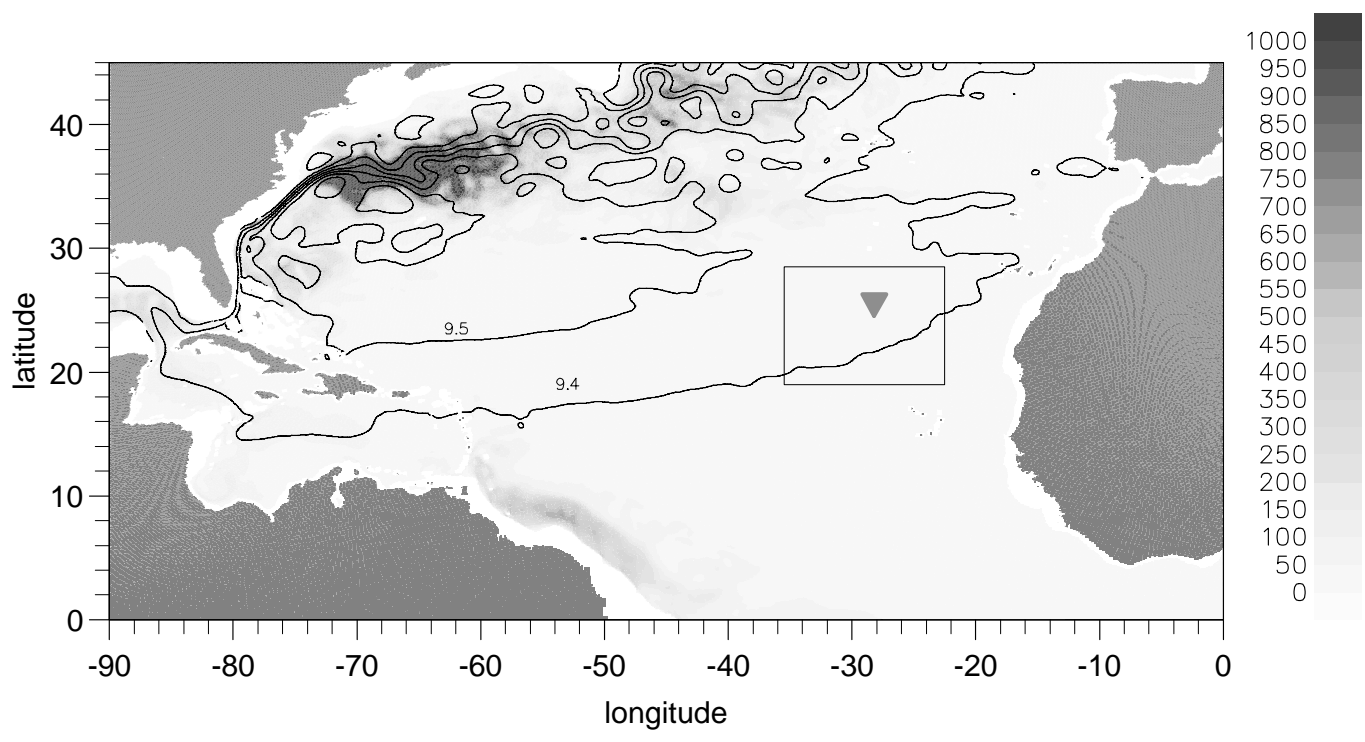

Figure 1: The shading gives the mean eddy kinetic energy $\left(\mathrm{cm}^{2} \mathrm{~s}^{-1}\right)$ at depth $314 \mathrm{~m}$ (model level 25) for year 1995 from the $1 / 12^{\circ}$ run. The highest eddy kinetic energy (indicated by darker shading) is in the region of the Gulf Stream. The contours (in units of $10^{4} \mathrm{~Pa}$ ) gives the mean pressure field at the same depth for the same year. The triangle indicates the site of tracer release in the model and the rectangular box corresponds to the plots shown in Figs. 2-5. 


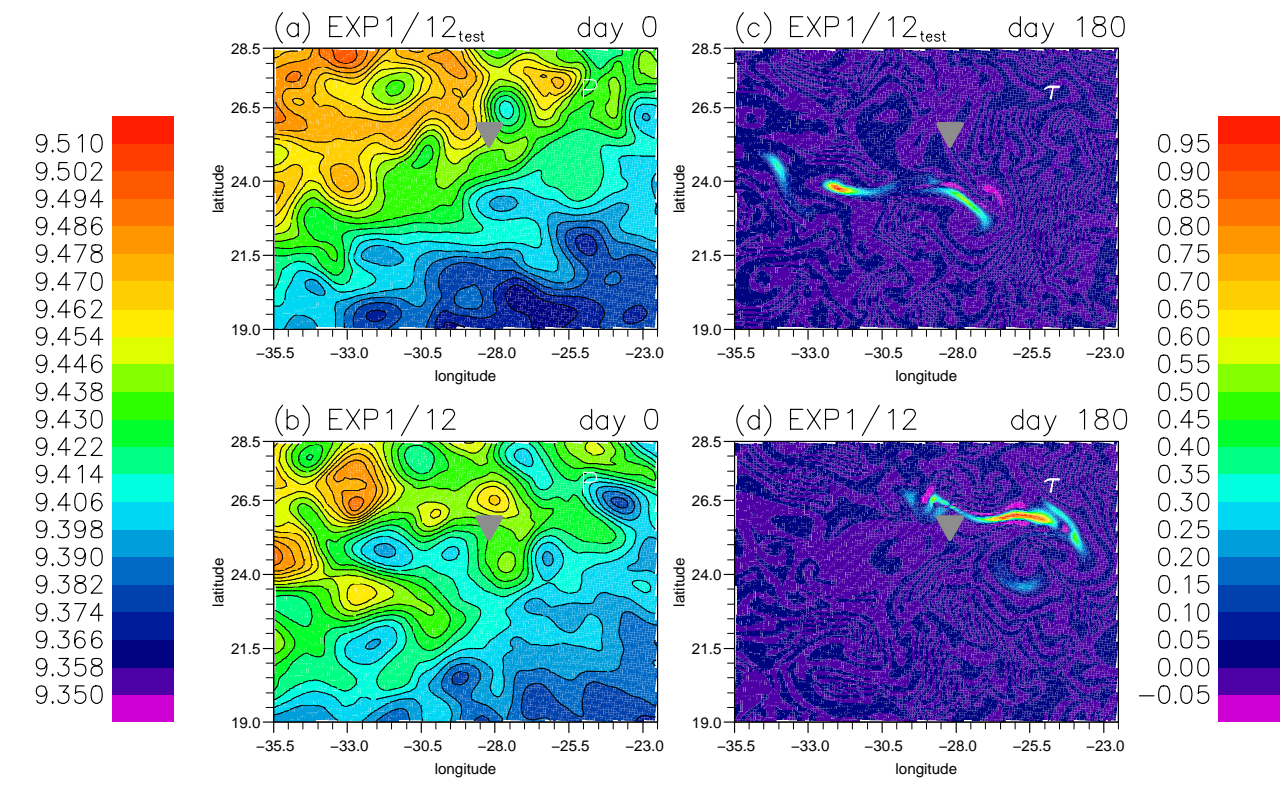

Figure 2: Five-day mean fields from the $1 / 12^{\circ}$ run. The left panels (a) and (b) are the pressure fields at $314 \mathrm{~m}$. The color scale is on the left with the units of $10^{4} \mathrm{~Pa}$ and contour interval is $80 \mathrm{~Pa}$. (a) is at the time of tracer release for EXP1/12 $2_{\text {test }}$. (b) is at the time of tracer release for EXP1/12. The right panels (c) and (d) are the tracer concentration on the target density layer after the 180 days (each right panel corresponds to the release on the left panel). The tracer concentration is normalised to have the maximum value 1 . The color scale is on the right with the magenta color showing the negative tracer value.
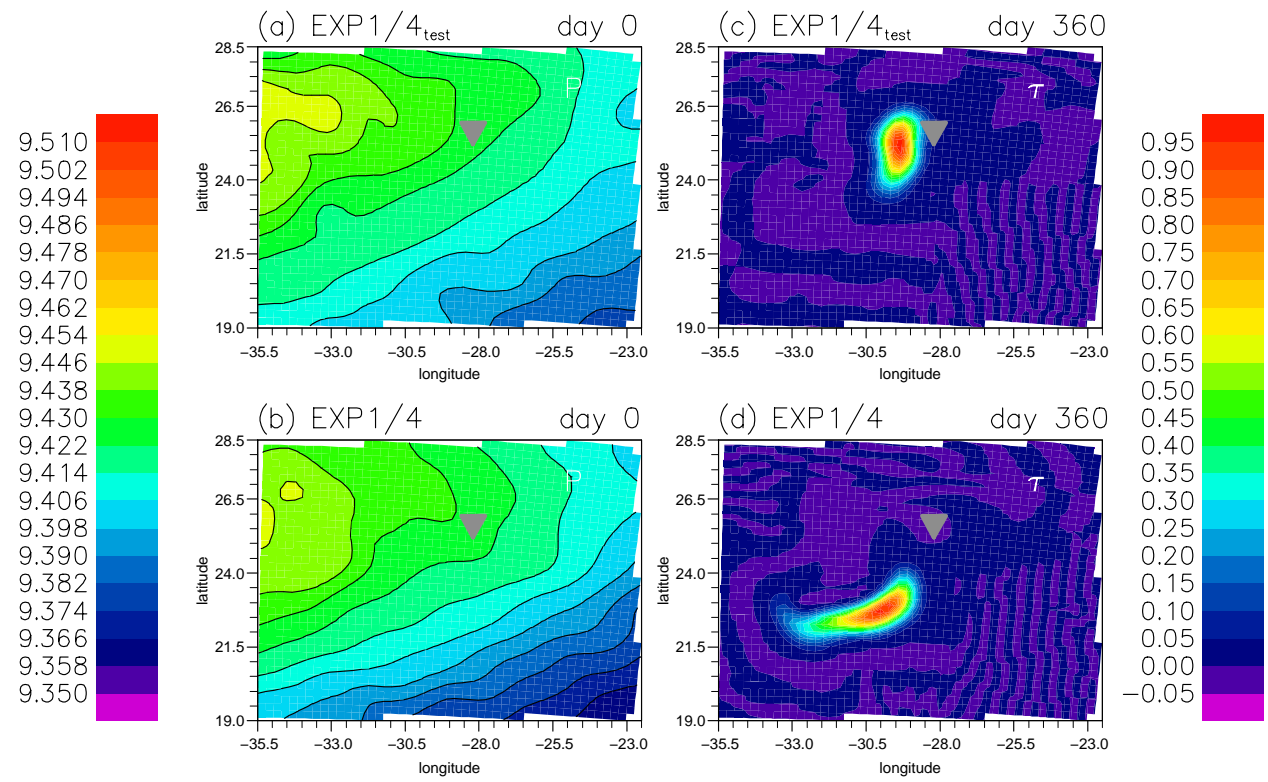

Figure 3: As in Fig. 2, but for the $1 / 4^{\circ}$ run. The tracer fields on the right panels are 360 days after releases. 
EXP $1 / 12$ day 180
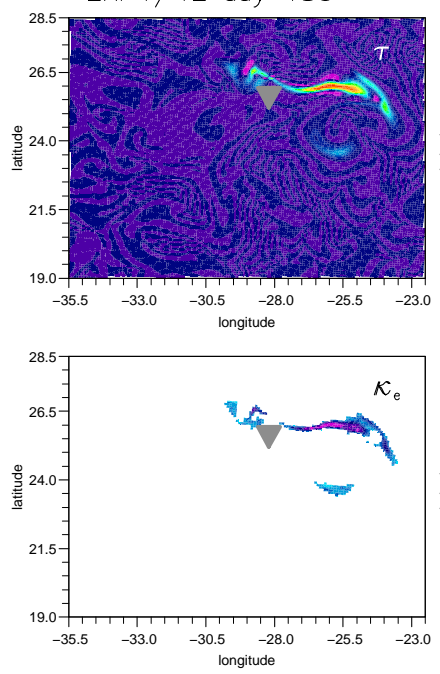

EXP $1 / 12$ day 365
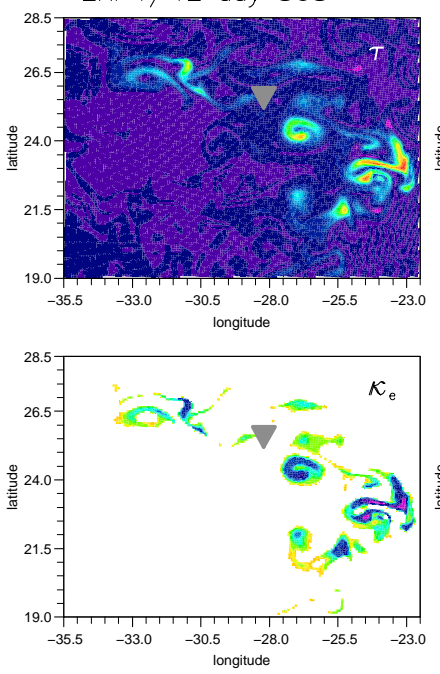

EXP $1 / 12$ day 730

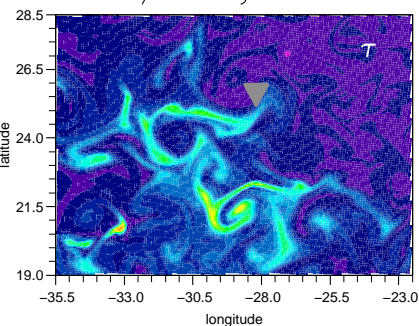

$\kappa_{\text {e }}$

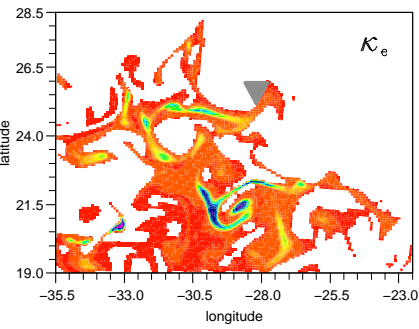

Figure 4: For EXP1/12. The upper panels are the normalised tracer concentration on the target density layer (color scale on the left). The lower panels are effective eddy diffusivity $\kappa_{e}\left(\mathrm{~m}^{2} \mathrm{~s}^{-1}\right)$ (color scale on the right). The left, middle and right panels correspond to day 180, 365 and 730 days, respectively.

EXP1/4 day 180
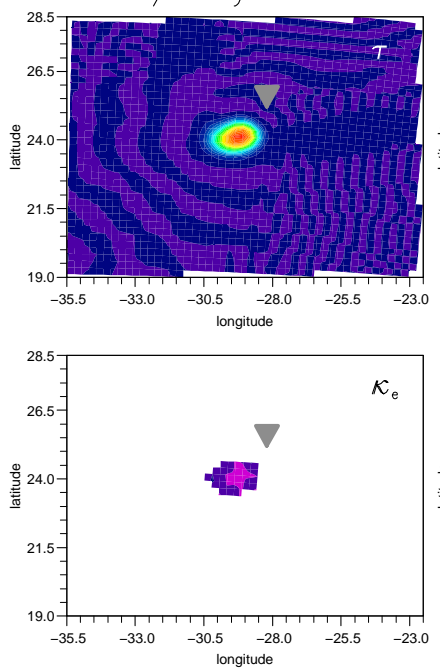

EXP1/4 day 365
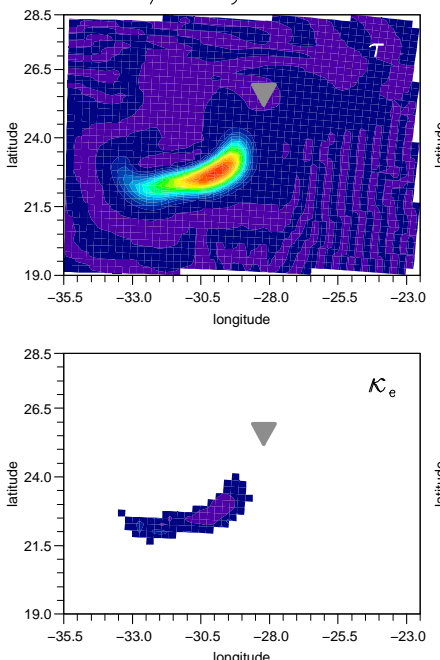

EXP $1 / 4$ day 730
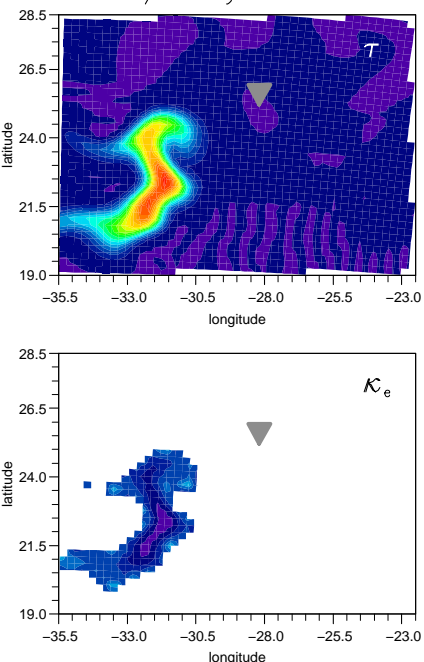

Figure 5: As in Fig. 4 but for EXP1/4. 


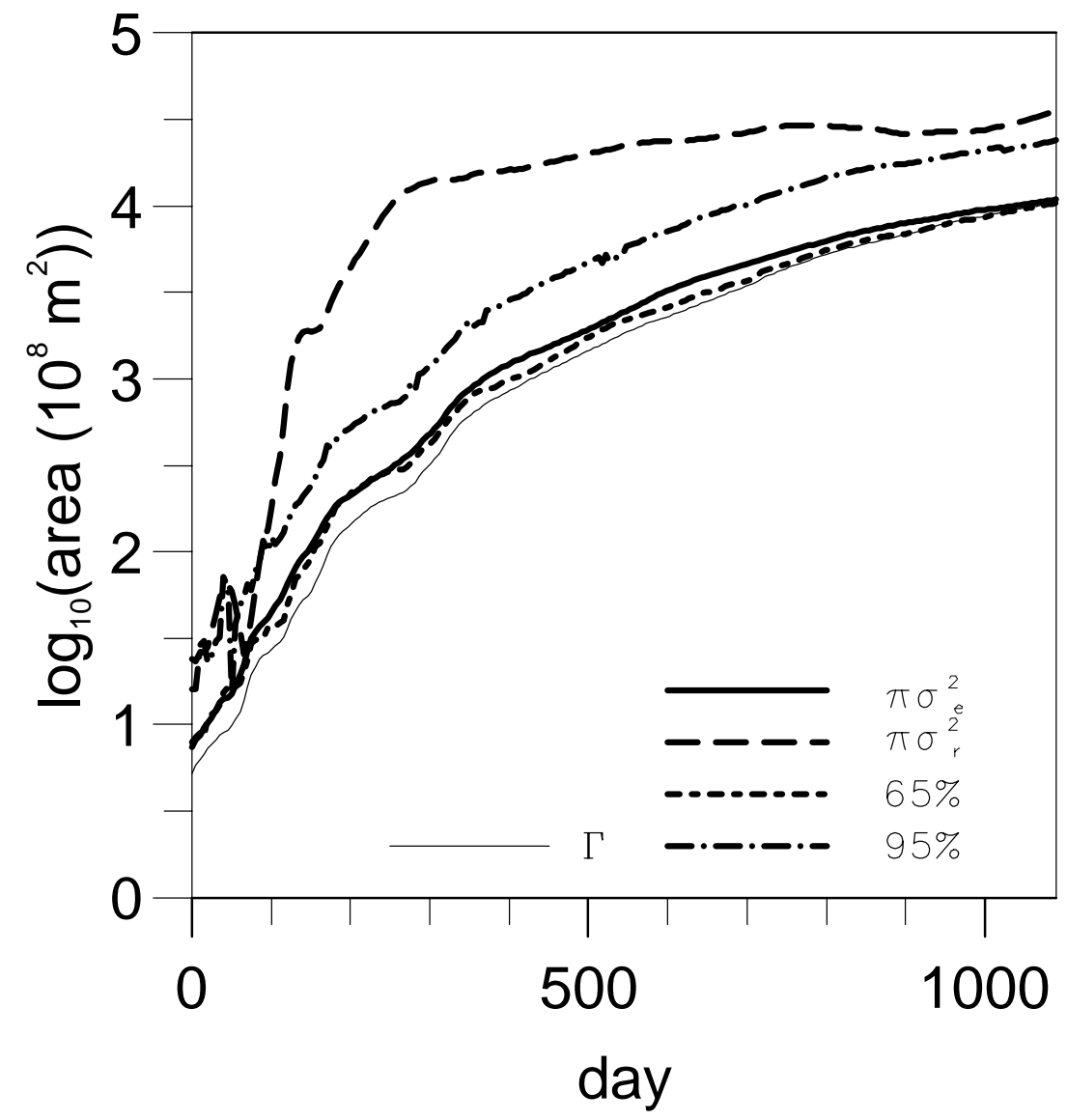

Figure 6: The three years time series of areas from EXP1/12: from the equivalent variance, $\pi \sigma_{e}^{2}$ (thick solid line), from the conventional distance variance, $\pi \sigma_{r}^{2}$ (dashed line), the tracer area containing $65 \%$ tracer load (dotted line), the tracer area containing 95\% tracer load (dot-dashed line), the tracer area, $\Gamma$ (thin solid line). 

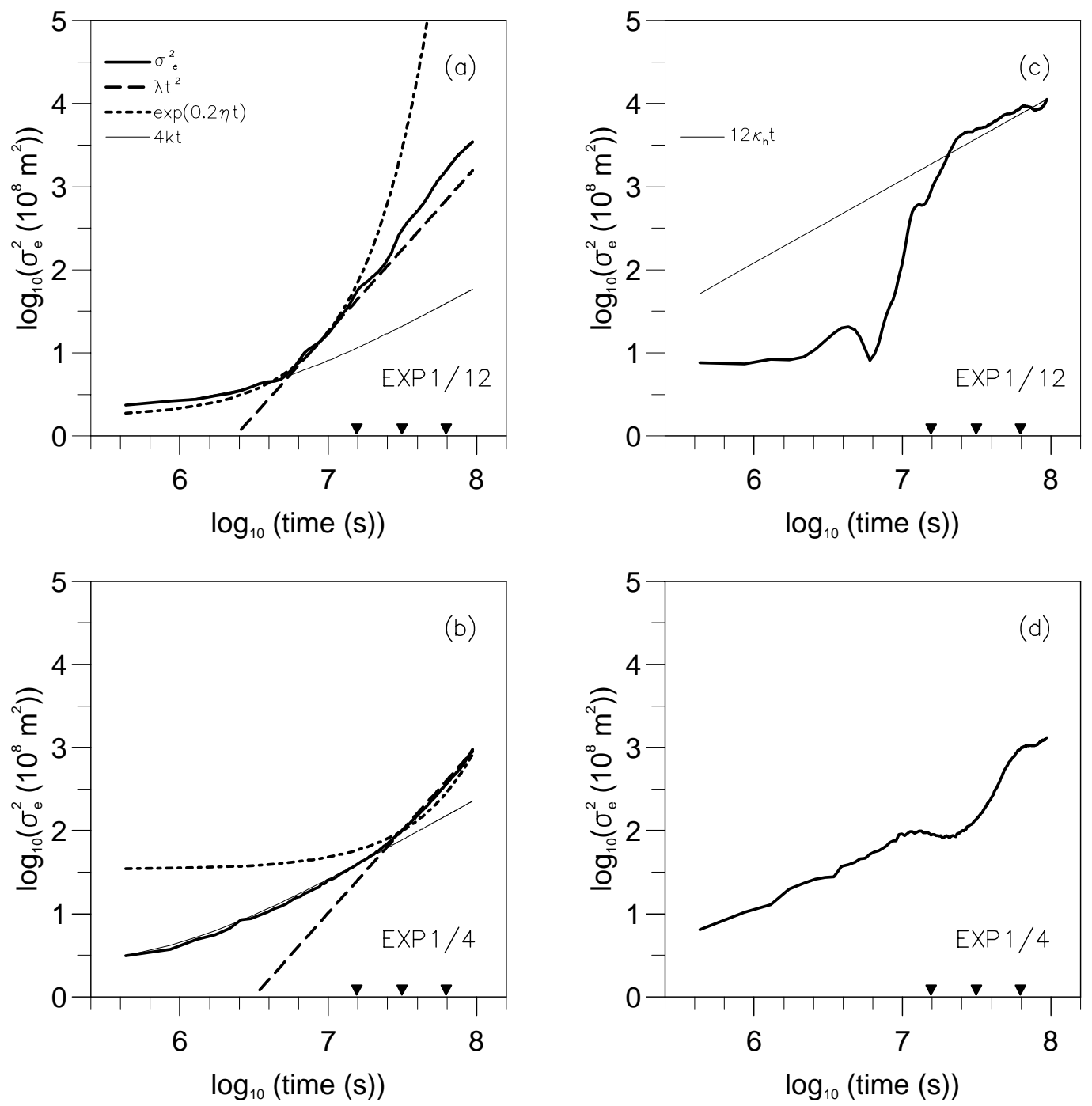

Figure 7: Left panels: the time series of the equivalent variance, $\sigma_{e}^{2}$ (thick solid lines), in log-log scale for the run of (a) $1 / 12^{\circ}$ and (b) $1 / 4^{\circ}$. The three triangles mark 180 days, 1 year and 2 years. The thin solid lines show $\sigma_{e}^{2}(0)+4 k t$ (where $k=15$ and $60 \mathrm{~m}^{2} \mathrm{~s}^{-1}$ in $1 / 12^{\circ}$ and $1 / 4^{\circ}$ run, respectively) (thin solid lines). The $\sigma_{e}^{2}(0)$ is the variance at the time of release. Note the thin and solid lines almost overlap. Also plotted are curves of the $2^{\text {nd }}$ power law, $\lambda k t^{2}$ (dashed lines), and the exponential law, $\sigma_{e}^{2}(T) e^{0.2 \eta(t-T)}$ (dotted lines), relevent to the stirring-dominated stage. $\sigma_{e}^{2}(T)$ is the value of $\sigma_{e}^{2}$ at the time $T$ (70 days and 360 days for $1 / 12^{\circ}$ and $1 / 4^{\circ}$ runs, respectively). See text for the values of straining rate $\lambda$ and stretching rate $\eta$. Right panels: the conventional distance variance, $\sigma_{r}^{2}$ (thick solid line). The superimposed line in EXP1/12 is $12 k_{h} t$, where $k_{h}=1000 \mathrm{~m}^{2} \mathrm{~s}^{-1}$ (thin solid line). 


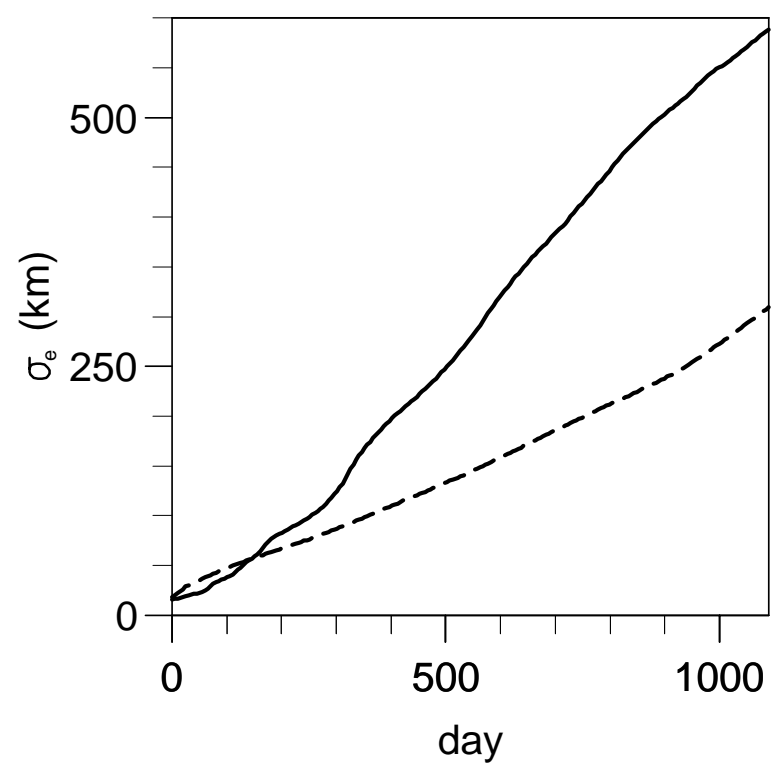

Figure 8: The time series of the equivalent deviation $\sigma_{e}$ from EXP1/12 (solid line) and EXP1/4 (dashed line).

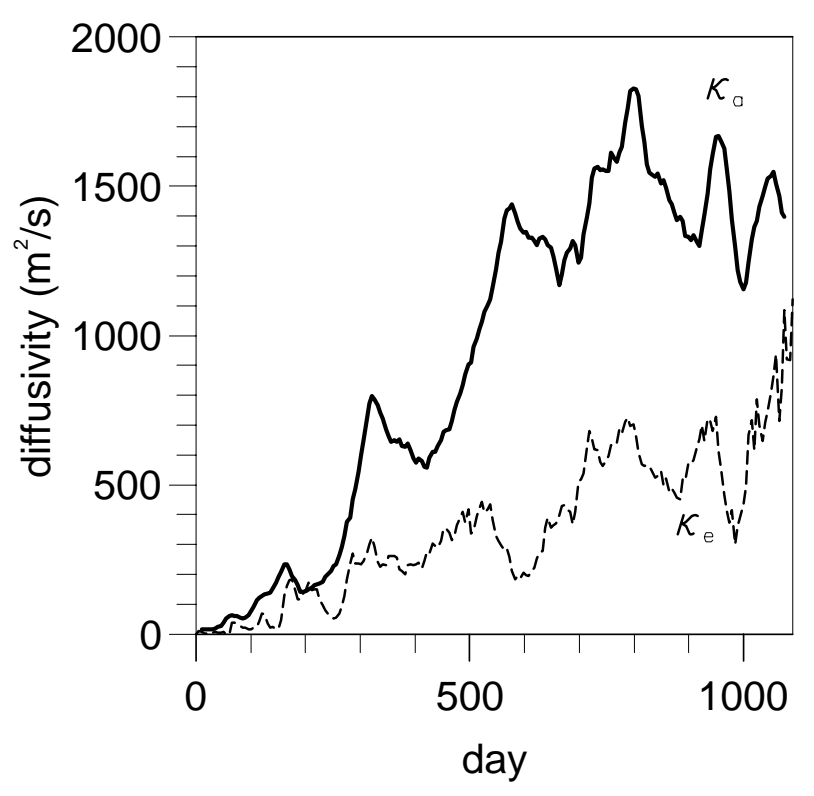

Figure 9: The time series of apparent diffusivity $\kappa_{a}$ (solid line) (smoothed with 30-day filter) and mean effective diffusivity $\bar{\kappa}_{e}$ (dashed line) from EXP1/12. 


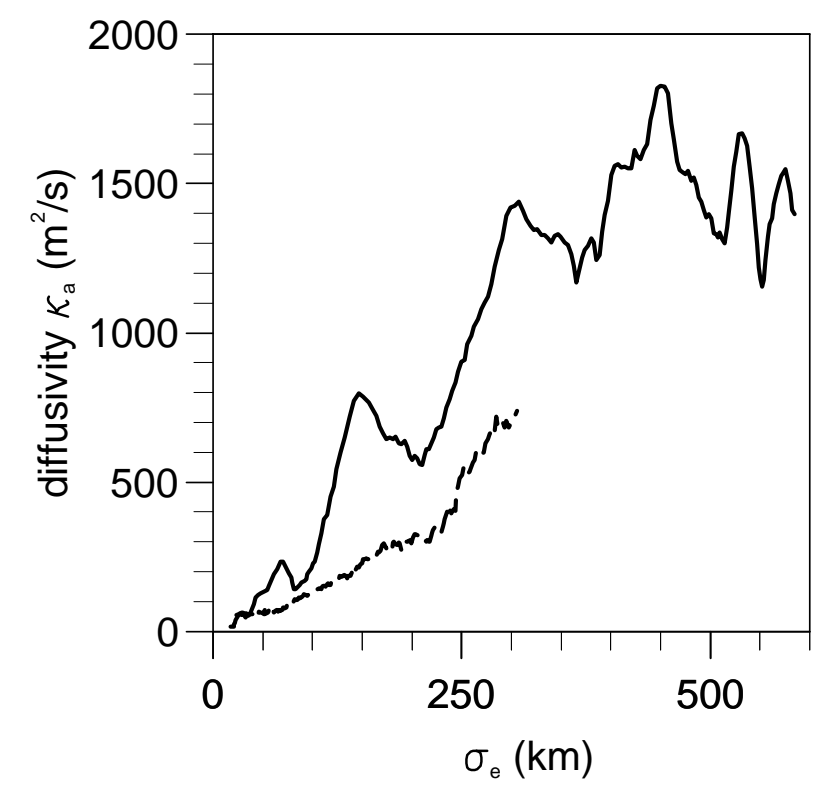

Figure 10: The time series of apparent diffusivity $\kappa_{a}\left(\mathrm{~m}^{2} \mathrm{~s}^{-1}\right)$ plotted as a function of equivalent deviation $\sigma_{e}$. EXP1/12 (solid line) and EXP1/4 (dashed line).
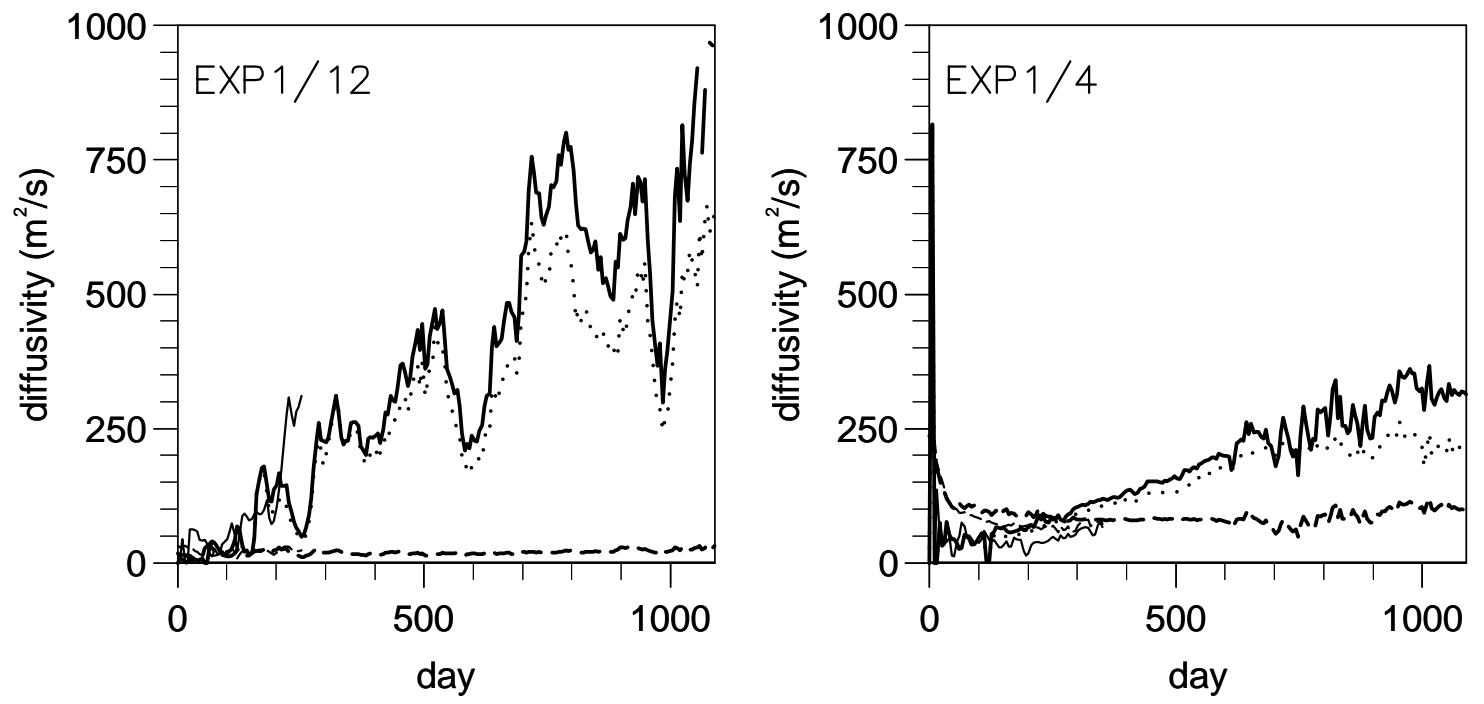

Figure 11: The diffusivity $\left(\mathrm{m}^{2} \mathrm{~s}^{-1}\right)$ over three years. The left panel is for the $1 / 12^{\circ}$ run and the right panel is for the $1 / 4^{\circ}$ run. The background diffusivity $k$ is given by the dashed lines and the mean effective diffusivity $\bar{\kappa}_{e}$ is given by the solid lines. The thick lines are from the main runs and thin lines are from the test runs. The dotted lines are $\bar{\kappa}_{e}^{\text {adjust }}=\bar{\kappa}_{e}+\bar{\kappa}_{e}^{S}$. 

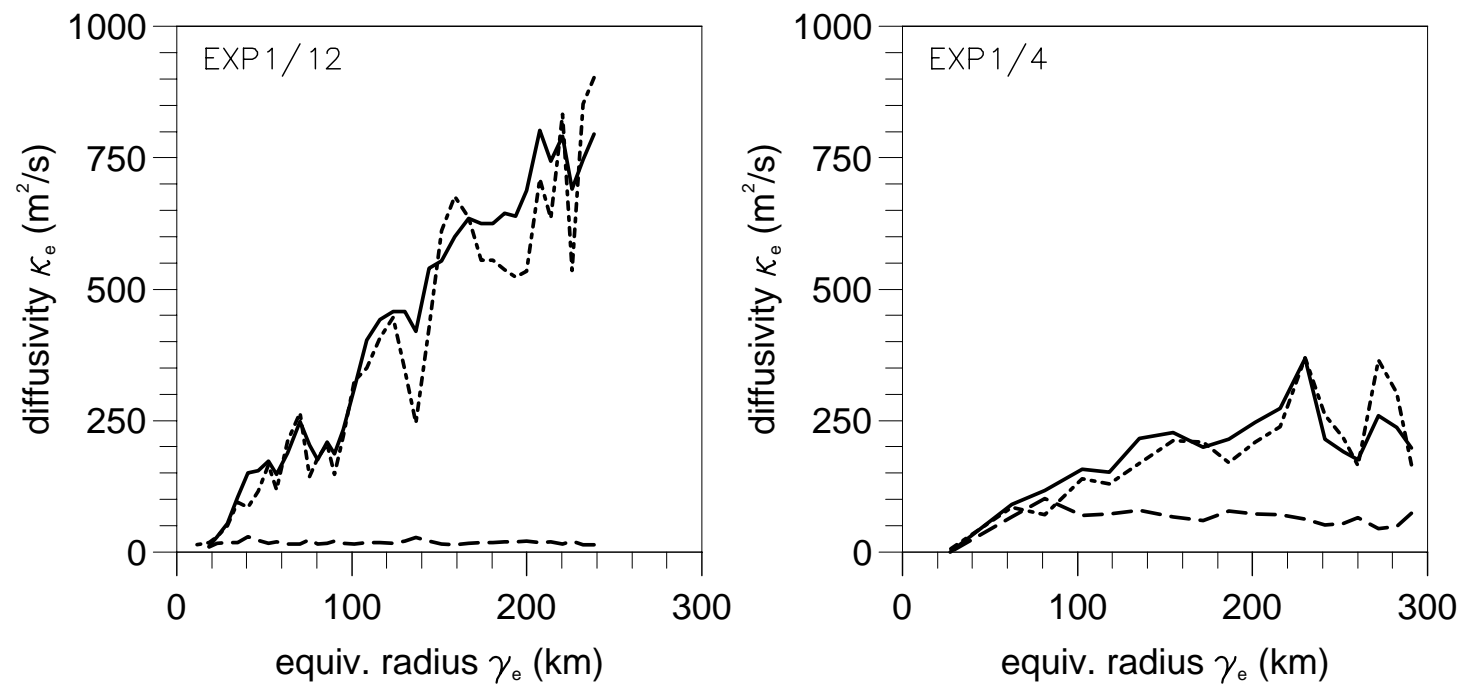

Figure 12: The diffusivity $\left(\mathrm{m}^{2} \mathrm{~s}^{-1}\right)$ as a function of equivalent radius $\gamma_{e}(\mathrm{~km})$. The dashed lines are the background diffusivities. The dotted line and the solid lines are the effective diffusivities calculated from (8) and (10), respectively. The left panel is from EXP1/12 at day 365 and the right panel is from EXP1/4 at day 730 . 


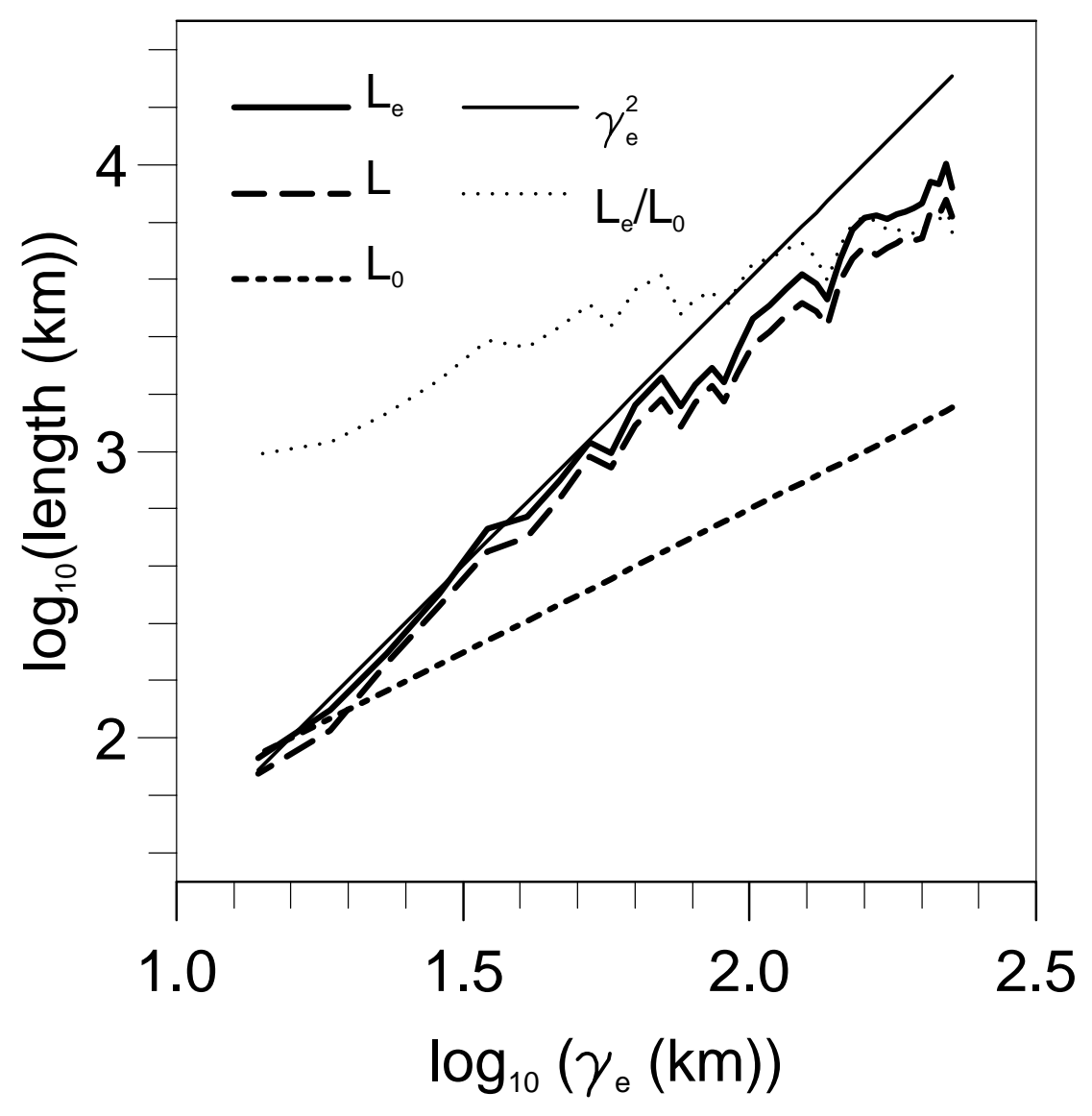

Figure 13: Length for the contours of tracer from EXP1/12 at day 365 as a function of equivalent radius $\gamma_{e}$ plotted in log-log scale: the equivalent length $L_{e}$ (thick solid line), the actual length $L$ (dashed line), the minimal length $L_{0}=2 \pi \gamma_{e}$ (thick dotted line). The superimposed lines are $\gamma_{e}^{2}$ (thin solid line) and $L_{e} / L_{0}$ (thin dotted line). 


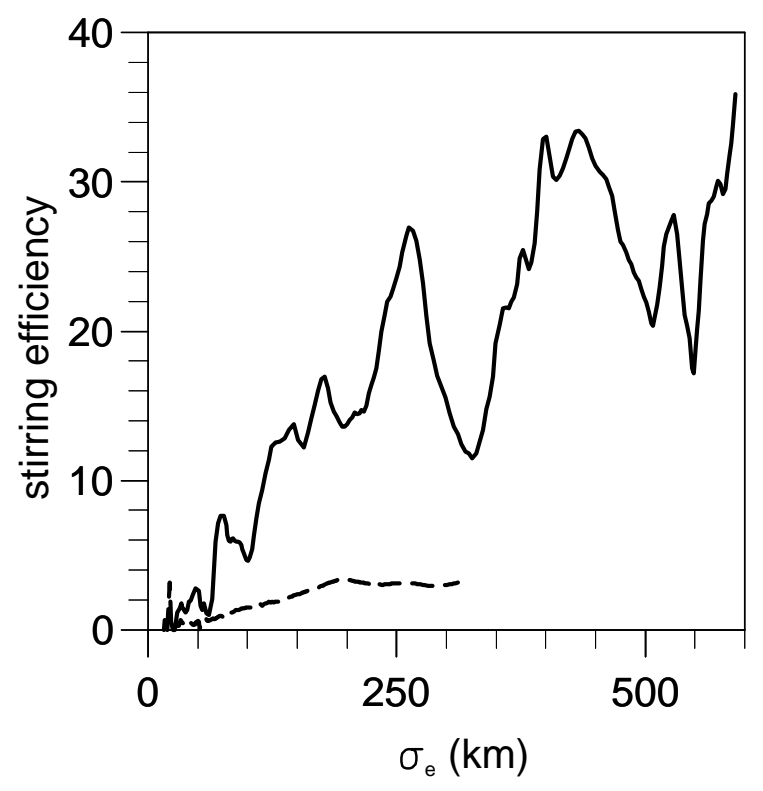

Figure 14: The eddy efficiency $\zeta$ plotted against equivalent deviation $\sigma_{e}$. The solid line is from EXP1/12 and the dashed line is from EXP1/4. 

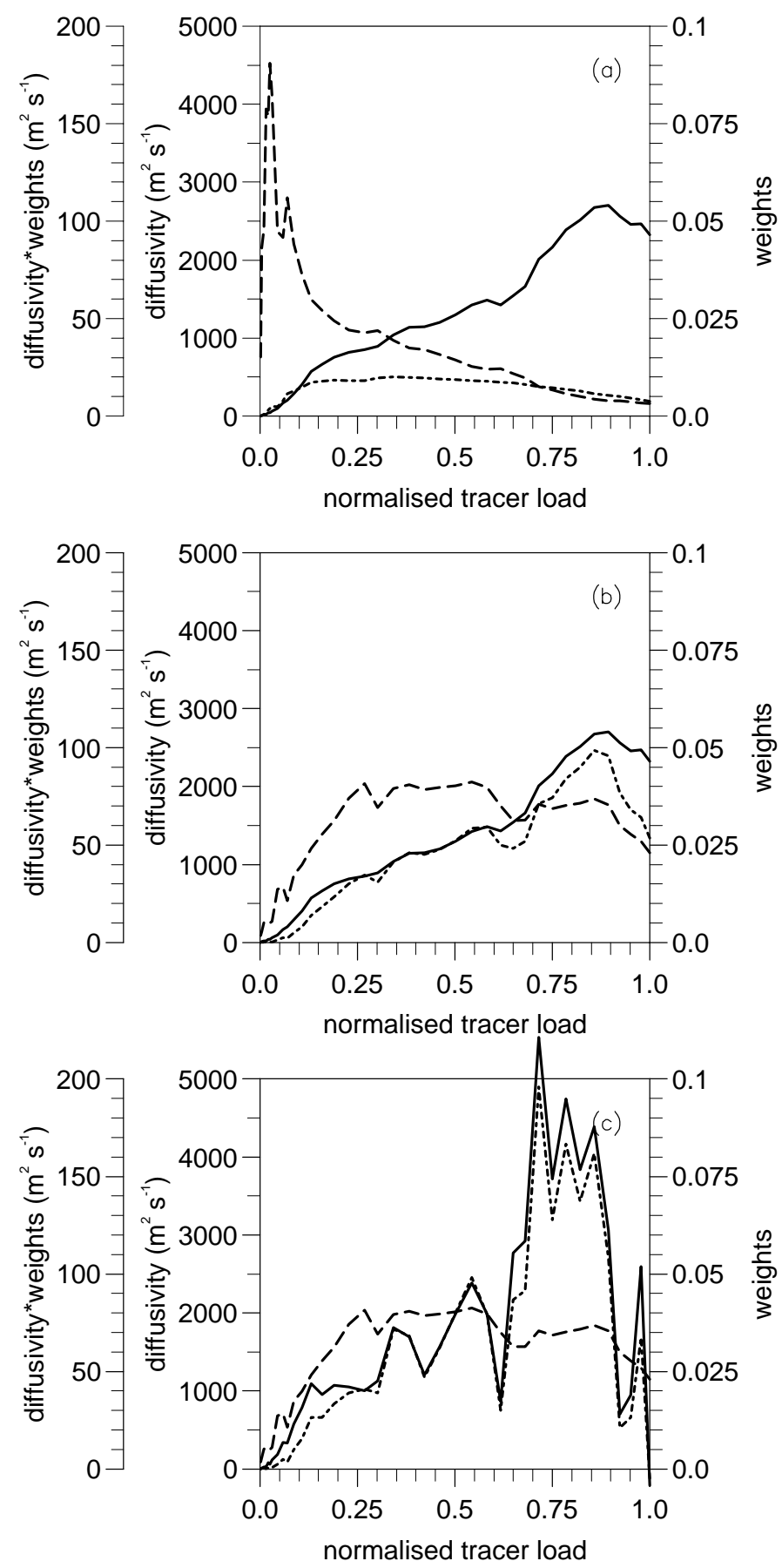

Figure 15: Quantities that contributes to mean diffusivities at day 730 of EXP1/12. (a) mean effective diffusivity $\bar{\kappa}_{e}$, (b) for comparison $\kappa_{s}=\int \kappa_{e} C d a / \int C d a$ and (c) apparent diffusivity $\kappa_{a}$. The $x$-axis is the normalised tracer load encompassed by tracer contours with 1 corresponding to total tracer load enclosed by the lowest tracer concentration. In (a), $\kappa_{e}$ (solid line), weighting $\left|\frac{\partial \hat{C}}{\partial \gamma_{e}}\right|^{2} d a / \int\left|\frac{\partial \hat{C}}{\partial \gamma_{e}}\right|^{2} d a$ (dashed line) and the multiplication of the two (dotted line). In (b), $\kappa_{e}$ (solid line), weighting $C d a / \int C d a$ (dashed line) and the multiplication of the two (dotted line). In (c), $\frac{\partial}{\partial \mathcal{A}}\left(\kappa_{e} \mathcal{A}\right)$ (solid line), weighting $C d a / \int C d a$ (dashed line) and the multiplication of the two (dotted line). 